\author{
Italique \\ Italique \\ Poésie italienne de la Renaissance
}

XII | 2009

Varia

\title{
Le poesie e le prose spirituali di Anton Francesco \\ Grazzini
}

\section{Franco Pignatti}

\section{(2) OpenEdition}

Journals

\section{Edizione digitale}

URL: http://journals.openedition.org/italique/228

DOI: 10.4000/italique.228

ISSN: 1663-4438

\section{Editore}

Librairie Droz

\section{Edizione cartacea}

Data di pubblicazione: 1 novembre 2009

Paginazione: 123-172

ISSN: 1423-3983

\section{Notizia bibliografica digitale}

Franco Pignatti, « Le poesie e le prose spirituali di Anton Francesco Grazzini », Italique [Online],

XII | 2009, online dal 01 novembre 2012, consultato il 19 avril 2019. URL : http://

journals.openedition.org/italique/228 ; DOI : 10.4000/italique.228 


$$
\text { Franco PignatTi }
$$

LE POESIE E LE PROSE S PIRITUALI D I A N T O F R A N C S C O GRAZZIN I 



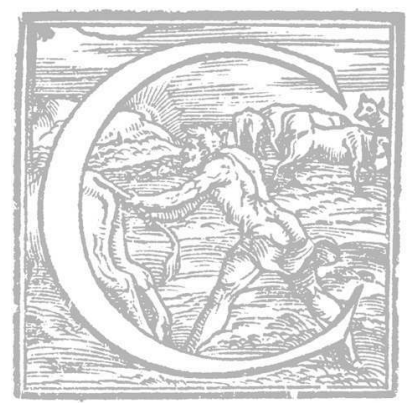

Hi si accinga a studiare le poesie religiose di Grazzini si trova dinanzi a un terreno non solo ancora da dissodare, ma del quale manca perfino un'idea dell'esatta estensione. In effetti, il profilo del Lasca come rimatore resta consegnato precipuamente all'identità di poeta burlesco, che già in vita prevalse e fu sancita dai contemporanei ("l'ottimo, e graziosissimo Lasca nostro, della giocosa poesia, e della berniesca piacevolezza, principalissimo erede rimaso ne' tempi nostri 》 lo proclamò il Salviati), ${ }^{1}$ oscurando altri ambiti in cui la musa grazziniana si espresse e a livelli non indegni, quello del sacro, per l'appunto, ma anche quello pastorale, che, fermo all'edizione delle Egloghe ed altre rime fornita da Gaetano Poggiali, ${ }^{2}$ pure attende una disamina critica chiarificatrice. Per il primo di questi due casi, che qui ci interessa, l'intento del presente saggio sarà dunque di delineare un quadro d'insieme coerente allo stato delle conoscenze, nonché di tentare un primo approfondimento degli aspetti che si presentano degni di particolare attenzione.

Il punto di partenza da cui prendere le mosse è la Tavola delle opere, in cui Grazzini traccio il bilancio della sua produzione letteraria al is settembre I566. Qui, alla rubrica Rime spirituali, sono registrate le seguenti composizioni: "Sonetti, da trenta. Madrigali, intorno a quindici. Capitoli, uno al Crocefisso. Stanze, la Festa di san Felice in Piazza, cioè l'Annunziazione della Vergine. Laude, da Quaranta ». Ad esse si devono aggiungere le quattro Commedie spirituali in prosa intitolate La croce, o Santa Helena, Santa Apollonia, Santa Caterina, Santa Orsola, e, alla rubrica Egloghe diece, l'egloga Passione, e morte del figliuol di Dio. Di tutto ciò, allo stato attuale delle ricerche, ci sono giunti $i$ componimenti tramandati dal manoscritto Magliabechiano 1029, cc. 202r-233r, autografo, ${ }^{4}$ per un totale di 39 sonetti, 4 ottave e l'egloga citata (Egloga decima, e ultima nella morte del Figliuol di Dio). ${ }^{5}$ Undici di questi sonetti, più un dodicesimo che nel Magliabechiano manca, sono testimoniati anche dal ben noto ms. II.IV.I della Biblioteca Nazionale Centrale di Firenze (cc. Ioor-Iosr), contenente le composizioni dei membri dell'Accademia degli Umidi. ${ }^{6}$ Entrambi i manoscritti furono utilizzati da Francesco Moüke per la sua edizione delle Rime grazziniane, che ospita tutte le poesie del Magliabechiano ad eccezione delle ottave e di un sonetto di contenuto anticlericale, mentre ha in più il sonetto supplementare del ms. II.IV.I e due madrigali. Un altro sonetto, assai modesto, si trova inserito nella seconda delle quattro Orazioni alla croce, edite nel I 822 da Domenico Moreni, ${ }^{8}$ che ricavò il testo di tre di esse dal Magl. XXXV.44, autografo, e per la quarta si servi della 
trascrizione eseguita da Gaetano Poggiali su un codice strozziano ed elargitagli dal figlio del Poggiali, Domenico.9 Il benemerito editore delle rime burlesche del Lasca, Carlo Verzone, identifico il manoscritto - non senza lasciarsi andare a un legittimo sarcasmo sulla diligenza impiegata dal Moreni nel ricercarlo ${ }^{10}$ - proprio nel Magl. VII.Io29, proveniente dalla biblioteca privata della famiglia Strozzi, nel quale l'orazione si trova intercalata alle rime alle cc. 226v-229v. Infine, tre sonetti del Magliabechiano diretti a Benedetto Varchi si conservano negli originali nel ms. Banco Rari 59 della Nazionale di Firenze.

Rispetto alla registrazione della Tavola delle opere, si noterà che, mentre la produzione lirica risulta abbastanza salvaguardata, o addirittura accresciuta, ad avere subito l'ingiuria del tempo sono soprattutto $i$ componimenti teatrali. La perdita non è di poco conto, dato che questi testi aurebbero permesso di vedere come Grazzini, autore (come è noto) quanto mai sensibile alle problematiche del linguaggio teatrale, si poneva rispetto a una tradizione molto radicata a Firenze, quella delle sacre rappresentazioni, caratteristica della religiosità quattrocentesca e ancora viva (come riscontra la diffusione tipografica) nel secolo successivo, ${ }^{\text {II }}$ ma che subi una rapida trasformazione sotto l'infusso del teatro comico profano. A queste forme teatrali, espressione di una fede popolare poco provvista dal punto di vista teologico, ma molto forte sul piano del sentimento comunitario e dei valori collettivi alla base della vita cittadina, sono da riportare le laude e le stanze composte per la festa dell'Annunciazione nella chiesa di S. Felice in Piazza, per la cui fattura si può azzardare le date del I533 e del I547, alle quali si ha notizia di solenni celebrazioni della festa. Nella prima data, in particolare, in occasione dei festeggiamenti per l'arrivo di Margherita d'Austria, futura sposa del primo duca di Firenze Alessandro, fu stampata, anonima, la Festa della Annuntiatione di nostra Donna di Feo Belcari (s.t., "Addi XXII. di Dicembre. Nel. I533") e, dopo un lungo periodo di abbandono, la rappresentazione fu messa in scena nella chiesa di S. Felice il I9, 20 e 21 dicembre, con largo concorso di folla. ${ }^{\mathrm{I}}$ La qualifica a parte di "commedie spirituali", con la specifica « in prosa», della Tavola delle opere indica composizioni di altro tenore, modellate sulla commedia antiqua, non farse in tre atti o rappresentazioni suddivise in quadri autonomi; è probabile che esse non fossero troppo distanti dalle commedie e farse «spirituali» che Giovan Maria Cecchi diede fuori, in versi, a partire da una certa data, ${ }^{\mathrm{I}}$ con il consapevole intento innovatore di rendere gli spettacoli cattivanti e leggeri adibendo $i$ ritrovati piacevoli del repertorio contemporaneo. Con ciò si veniva incontro alle esigenze di un pubblico che, scaltrito dalla consuetudine con la moderna scrittura teatrale, non si accontentava più delle forme incondite e popolareggianti in cui si era espresso finora il teatro sacro, e neppure era disposto a recarsi a teatro 
per farsi edificare con ingenui stratagemmi spettacolari o tirate moraleggianti. A parte la registrazione del I566, la notizia che Grazzini avesse già al suo attivo rime sacre a una data di parecchio anteriore si ricava dal noto episodio che il poeta affidò al sonetto rinterzato Ascoltate di grazia, Bettin mio, diretto al mercante fiorentino residente a Roma Bartolomeo Bettini: ${ }^{14}$ quando Vittoria Colonna si trovava a Firenze per ascoltare le prediche di Bernardino Ochino, riferisce Grazzini, alcuni sonetti scritti da lui circolarono sotto il nome della marchesa di Pescara, riscuotendo un generale apprezzamento. ${ }^{15}$ Il passo merita una citazione integrale:

Voi dovete saper, che quella chiara fra l'altre donne più che in cielo il sole gloriosa Marchesa di Pescara venne in Fiorenza, acciò l'alte parole del Scappuccino udisse, onde s'impara come Dio s'ama veramente e cole.

Udite, e non son fole, che dopo Pasqua la prima giornata assai per tempo in santa Reparata, dove molta brigata stava lieta aspettando con amore il tanto dotto e buon predicatore, quando del seno fuore certi sonetti si trasse un mio amico fatti da me si può dire ab antico.

Egli è quel ch'io vi dico, che domandato di chi era l'impresa, disse a caso colui: della Marchesa.

Ma tosto che fu 'ntesa la voce intorno di cotal novella, subito gli fu fatto capannella.

Questa, Bettino, è bella, che un uom d'autorità, che presso gli era, gli prese in mano, e con gran sicumera cominciò di maniera lodando a dir di sonetto in sonetto: oh questo è bello! oh quest'altro è ben detto!, ch'ognun fu lì costretto, poi che fur letti, per la meraviglia serrar le labbra ed inarcar le ciglia.

E molto si bisbiglia del gran valor, dell'alta sua dottrina, tutti dicendo, ch'ella era divina. 


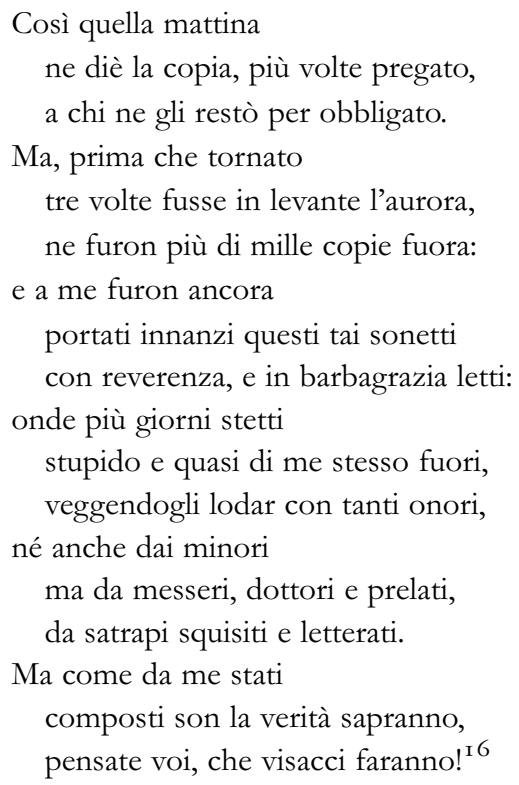

Trascurata dagli storici, la testimonianza è così puntuale da richiedere una ricognizione sulla biografia della Colonna per verificare se concordi con essa. Nel marzo 1538 Bernardino Ochino predicò a Pisa, proveniente dagli stati estensi, dove aveva dimorato a lungo a Ferrara, ospitato da Ercole II e Renata di Francia. ${ }^{17}$ Si trattava di un cambio di programma rispetto agli accordi presi già l'anno precedente con la vedova di Alessandro de' Medici Margherita d'Austria, secondo i quali il cappuccino avrebbe dovuto recarsi a Firenze. Del cambiamento fu informata per lettera Vittoria Colonna, che, dopo avere soggiornato anch'ella a Ferrara, dal maggio dell' anno precedente, $e$ avervi coltivato il disegno di recarsi in Terrasanta muovendo da Venezia, il 22 febbraio 1538 lascio la capitale estense alla volta del ducato di Toscana. La marchesa di Pescara si diresse dunque a Pisa e vi ascoltò l'Ochino finché la presenza del frate non fu reclamata a Firenze: il 26 marzo, ragguagliando Ercole II dei suoi imprevisti spostamenti, scriveva che il cappuccino era partito per Firenze il giorno prima ed ella attendeva il momento opportuno per trasferirsi a Lucca, ai bagni. ${ }^{18}$ Già il I8 marzo, infatti, gli Anz̧iani di Lucca, appreso che la gentildonna intendeva soggiornare nella loro città, avevano inviato una rappresentanza a porgerle omaggio e la Colonna aveva risposto il 22 marzo. ${ }^{19}$ Dalla missiva all'Este la partenza non sembra imminente né la destinazione avere risvolti diplomatici, ma lo spostamento fu probabilmente accelerato dal fatto che per Lucca doveva transitare Paolo III, diretto a Nizza per l'incontro tra l'imperatore e il re di Francia. La Colonna si trovava perciò a Lucca già ai primi di aprile, e porse il suo omaggio al 
pontefice, che sostò nella città toscana dal 6 all'8 del mese; ${ }^{20}$ il 9 scrisse dell'incontro al duca di Ferrara. ${ }^{21}$ Ma poco dopo lasciò la città, dove fece ritorno in maggio, in compagnia di un'altra donna della Riforma italiana, come lei fervente sostenitrice dei cappuccini, la duchessa di Camerino Caterina Cibo. Il secondo arrivo della Pescara a Lucca è compreso tra il $13^{22}$ e il I 8 maggio, data di una nuova missiva ad Ercole II; nella città toscana la gentildonna rimase fino all'autunno, avendo nuovamente occasione di ascoltare l'Ochino predicare nell'agosto successivo. ${ }^{23}$ Tirando le somme, a Lucca la Colonna trascorse in massima parte il suo soggiorno in Toscana nel I538, come ella stessa ebbe a precisare in una lettera all'Aretino il 25 settembre: "De Luca, ove son stata sempre, non a Pisa, come dice la vostra, ma passai de li "); ${ }^{24}$ ma, in questa ricostruzione degli spostamenti della marchesa di Pescara bisognerà incasellare la testimonianza di Grazzini, la quale si colloca nell'intervallo tra la prima e la seconda presenza lucchese della gentildonna. Dopo l'incontro con Paolo III, impaziente di ascoltare i sermoni della sua guida spirituale, ella non seppe restarsene inattiva e si spostò nella capitale medicea; nel I538 Pasqua cadde il 21 aprile e l'episodio raccontato da Grazzini avvenne il giorno di Pasqua dell' Angelo: dunque le date concordano. Di questo passaggio fiorentino della Colonna la poesia grazziniana costituisce l'unica prova diretta, se si eccettuano i costituti del secondo processo di Pietro Carnesecchi dinanzi al Sant'Uffizio, il quale, interrogato in due occasioni, a breve distanza di tempo, sui suoi rapporti con la Pescara, ricordò gli incontri a Lucca, ai bagni, nel I538 e di averla vista nello stesso anno a Firenze, quando la marchesa vi si trovava di passaggio, appunto per recarsi ai bagni; naturalmente tacque in entrambe le circostanze sulle prediche del frate apostata, all'origine degli spostamenti della Colonna in questa stagione. ${ }^{25}$

Il sonetto di Grazzini fu di certo composto a ridosso degli avvenimenti, come fa pensare la struttura di missiva in versi e il tono di accalorata relazione dei fatti che lo caratterizza. Oltre a fornire uno squarcio prezioso sulla produzione poetica del suo autore, esso ci parla altresi dell'esistenza a Firenze di un vero e proprio orizzonte di attesa per le rime della Colonna dal carattere francamente essoterico, esterno al circuito selezionato delle frequentazioni intellettuali che costitui il primitivo circoscritto uditorio della gentildonna poetessa, notoriamente ritrosa a divulgare esemplari dei suoi esercizi poetici. Attenzione, quella certificata da Grazzini, che trova riscontro di li a poco nell'edizione fiorentina delle Rime, ad istanza di Niccolò Zoppino, del luglio I539, e in seguito nell'interesse mostrato per la poetessa dai letterati dell'Accademia Fiorentina, che la inclusero tra gli autori moderni oggetto delle loro lezioni accanto al grande archetipo petrarchesco. ${ }^{26}$ Alla sua scomparsa, il 25 febbraio I547, Grazzini compose un sonetto che è tra le cose più toccanti delle sue poesie religiose: 


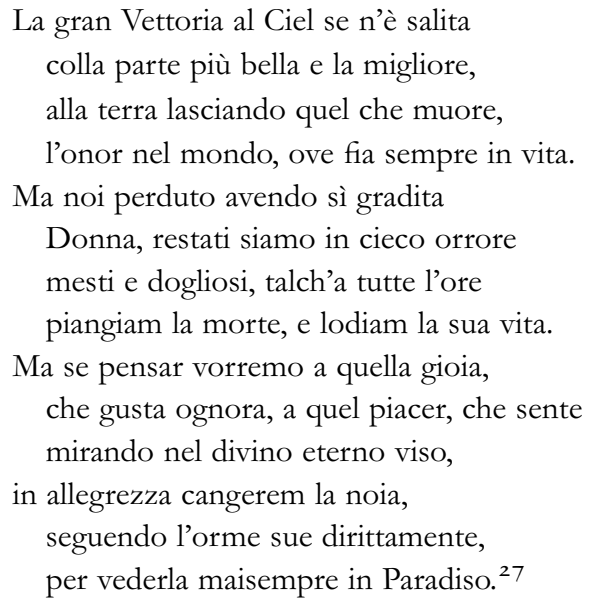

Peraltro, secondo la cronologia ricostruita da Alan Bullock, il I538 segna uno snodo importante nella maturazione della poetessa, dato che coincide con il trapasso dalla fase dominata dal tema amoroso a quella in cui comincia ad affacciarsi lo stimolo religioso, destinato a divenire predominante a partire dal I540. Nella prima raccolta a stampa delle Rime, l'edizione parmense del I538 (in cui, sia detto per inciso a proposito della circolazione di apocrifi, sono incluse nove poesie di altri autori), sono presenti solo alcuni sonetti spirituali, e appena dalla ricordata edizione fiorentina dell'anno successivo la dicitura "Sonetti spirituali » (in numero di IO) comincia ad apparire sul frontespizio. ${ }^{28}$ Dato che $i$ sonetti, di cui parla Grazzini, sono certamente sonetti spirituali, l'aneddoto attesta che la nuova maniera della poesia della Colonna era nota a Firenze, o per lo meno esisteva per essa un'aspettativa diffusa, tale da dare luogo all'equivoco raccontato al Bettini.

Ma venendo a Grazzini, anche se il sonetto conclude in tono per noi meno interessante, traendo la morale che il giudizio comune sulle opere letterarie dipende dalla fama della persona cui vengono attribuite e che l'opinione governa il mondo in vece della verità, non si può fare a meno di chiedersi se il caldo elogio tributato al predicatore cappuccino sia un apprezzamento generico, ovvero sia il segnale di un più sostanziale accostamento alla problematica religiosa nell'ottica dell'evangelismo italiano. Inclino per la prima ipotesi, e cioè che quanto Grazzini esprime qui non vada oltre $i$ sentimenti di commossa partecipazione che $i$ sermoni ochiniani universalmente suscitavano negli ascoltatori fino a livelli di autentico parossismo. ${ }^{29}$ Resta tuttavia il fatto che su di lui agi in qualche misura la predicazione ochiniana, e a quella data le sue poesie spirituali erano tali da ingenerare la confusione lusinghiera con quelle di uno degli esponenti più illustri degli ambienti filoriformati. Una ricognizione degli scritti religiosi di Grazzini alla luce dell'ipotesi che su di essi abbiano 
esercitato qualche suggestione, almeno per un momento, tesi vicine alla Riforma si impone come obbligatoria.

Le Orazioni alla croce furono composte da Grazzini per la ricorrenza del Venerdi santo e pronunciate presso qualche confraternita laicale, di cui $i$ codici tacciono il nome come pure le date, in cui vennero presentate. Grazzini prendeva parte alle attività della Compagnia della Cicilia, che aveva la sua sede principale nel chiostro di S. Maria Novella sotto il titolo di S. Lorenzo in Palco, ma si riuniva in un oratorio a Fiesole per festeggiare l'Annunciazione e la festa della santa (22 novembre). In essa presentò una «Oratione exortatoria recitata per uno Romito nella Compagnia della Cicilia di Fiesole, l'anno I540. Fatta per vincere e Notai col sospendere uno capitolo ", trasmessa dal ms. II.IV.I della Nazionale di Firenze subito di seguito ai Sonetti spirituali, ${ }^{30}$ e sette canti carnascialeschi recanti nei codici la rubrica « andato alla Cicilia ", il secondo anche la data I543. ${ }^{3 \mathrm{I}}$ La stessa destinazione, o in qualche altra compagnia laicale di stampo religioso, avranno avuto le commedie spirituali. ${ }^{32}$ Probabilmente Grazzini era affiliato anche alla Compagnia di S. Domenico, detta del Bechello (da Beco, forma popolare per Domenico), alla cui attività partecipavano diversi letterati fiorentini, tra cui lo Stradino, ${ }^{33}$ Giovambattista Gelli, ${ }^{34}$ il Bientina; soprattutto, il Venerdi santo del I 549 Benedetto Varchi vi pronunciò il suo Sermone alla croce, considerato da Paolo Simoncelli una " cripto-ristampa del Beneficio di Cristo $»^{35}$ per le riprese letterali di passi del libello evangelico sulla teoria della giustificazione per fede.

Anche il testo delle Orazioni presenta forti affinità con le tesi riformate per quanto attiene alla dottrina della giustificazione per fede, quale si ricava dal Catechismo valdesiano e dal Beneficio di Cristo, oltre che dagli scritti ochiniani, anche se è difficile indicare riprese puntuali di questi testi. ${ }^{36}$ Del resto, Grazzini si muove a un livello differente: le Orazioni sono opere devozionali, inserite in una precisa cornice liturgica, non scritti dottrinali come quelli del Valdés e di Benedetto da Mantova o un'omiletica militante e di grosso spessore teologico come $i$ sermoni ochiniani, gli uni e gli altri scritti impegnati a persuadere il destinatario e ad oppugnare le tesi avverse con una solida strumentazione argomentativa e scritturale. Nelle Orazioni grazziniane il registro controversistico lascia spazio a un tono di appassionata parenesi, basata sulla convenzione che gli ascoltatori siano portati a condividere gli argomenti esposti dall'oratore e che un comune sentimento religioso unisca entrambi sul modo di concepire la fede. L'operazione compiuta da Grazzini di rivolgersi lui, laico senza una specifica preparazione teologica, a un uditorio di laici, che si intuisce vicino a lui per estrazione sociale, convinzioni e stile di vita, non è troppo distante da quella attuata nel Is49 dal Varchi, ma in quel caso a un livello più raffinato, da parte di un letterato 
perfettamente consapevole dei suoi mezzi intellettuali e retorici, così come della posizione indipendente che si era conquistato (o si era illuso di avere conquistato) nell'establishment culturale mediceo. Varchi era in grado di elaborare una raffinata costruzione oratoria in cui infiltrare abilmente proposizioni eterodosse attingendo in via diretta a un testo come il Beneficio, che era ormai ufficialmente fuori dalla dottrina della Chiesa da quando il concilio di Trento con il Decretum de iustificatione del 13 gennaio I547 aveva sancito la condanna della dottrina protestante della giustizia imputata. ${ }^{37}$ Come ha illustrato Simoncelli, siamo in presenza di una strategia nicodemitica, che, in un contesto che non consentiva di professare apertamente il proprio credo, ambisce a conciliare l'adesione alle tesi evangeliche con l'ossequio formale alla Chiesa, dinanzi a un pubblico in grado di comprendere e decrittare questa tattica dissimulatoria. ${ }^{38}$ Nelle Orazioni grazziniane, al contrario, l'approccio alla tematica è diretto e immune da strategie di mascheramento e reticenza. Quando, in un caso, Grazzini mostra di voler rientrare sul terreno dell'ortodossia, lo fa in maniera tanto brusca da sembrare insincera, ovvero da suscitare nel lettore il dubbio che le tesi esposte prima con tanto fervore riflettano in realtà un'attenzione blanda alle problematiche teologiche, passibile di rapidi ritorni all'ordine, ove se ne avverta l'opportunità. Nella chiusa della quarta orazione l'invito ad adorare il crocifisso e l'invocazione della grazia terminano con un richiamo alla funzione di guida della Chiesa (nominata solo qui nelle Orazioni) e all'importanza delle opere per la salvezza eterna che contraddice la dottrina della giustificazione e l'idea ecclesiale esposta fin li: ${ }^{39}$

Non badate, non tardate più, venitene oramai, Fratelli e Padri dilettissimi; venitene oggi a chieder grazia con tutto il cuore a colui, che v'aspetta colle braccia aperte, pregandolo oggi divotamente, che vi doni Fede viva, Speranza certa, e Carità perfetta, e che vi accenda sopra tutto, arda ed infiammi il cuore del suo santissimo amore; acciocché seguendo la Santa Madre Chiesa, e obbedendo a' suoi comandamenti, il più che sia possibile, e facendo le buone operazioni colla sua grazia, in virtù principalmente del Sangue sparso, e per li meriti ella sua asprissima Passione; alla fine della vita vostra possiate godere i frutti di quella, e le sue santissime promessioni. ${ }^{40}$

Questo richiamo all'ordine è anche l'unico caso in cui il testo delle Orazioni, altrimenti avaro di particolari utili a questo fine, offre un appiglio per un'ipotesi di datazione, oltre quel limite del I547, al quale, a parte la definizione dottrinale del Tridentino di cui si è testé detto, si assiste a una decisa stretta da parte di Cosimo I alla linea di tolleranza religiosa osservata fino a quel momento: ${ }^{41}$ il I548, per citare un solo episodio eclatante, è l'anno del processo a Ludovico Domenichi per la traduzione dei Nicodemiana di Calvino. ${ }^{42}$ Da questo momento, come ha sostenuto Simoncelli, ${ }^{43}$ si registra l'intensificarsi 
della circolazione occulta del Beneficio negli ambienti letterari fiorentini: lo scritto di Benedetto da Mantova, rivisto e rielaborato da Marcantonio Flaminio, era un testo che senza alcuna possibilità di attenuanti si identificava con le posiżioni riformate, e perciò, in una situazione in cui l'unità del mondo cristiano era ormai definitivamente compromessa, poteva circolare soltanto per vie coperte e in cenacoli ristretti privi di risonanza sociale, proprio il contrario del linguaggio e delle modalità comunicative che caratterizzano le Orazioni grazziniane. Tranne che per l'ultima di esse, credo dunque che ci si debba attestare per le date di composizione ai primi anni Quaranta. ${ }^{44}$

Entro il quadro d'insieme, che si è delineato, è agevole rilevare le peculiarità che distinguono le Orazioni tra loro. La prima è quella che più è vicina agli schemi dell'omiletica tradizionale, di cui ripropone l'esegesi allegorica di passi veterotestamentari considerati come annunci della croce. Dopo avere aperto con la citazione delle parole di Gesù ai discepoli di Emmaus sulla necessità del martirio ("Non doveva forse il Cristo patire tutto questo ed entrare nella sua gloria? ", Lc 24, 26), Grazzini affastella come figurae crucis l'arca di Noè, la scala che dalla terra sale in cielo sognata da Giacobbe (Gn 28, I2), la visione della gloria di Dio avuta da s. Stefano durante la lapidazione (At 6, 55-60), l'albero di ginestra sotto cui si riposò il profeta Elia (I Re 19, 4), il cedro all'ombra del quale si siede la sposa nel Cantico dei cantici $(2,3)$, la pietra su cui Giacobbe posò il capo per dormire (Gn 28, Io e I8), il sangue del capro con cui i fratelli tinsero le vesti di Giuseppe per mostrarle al padre ( $\mathrm{Gn}$ 37, 3I), il legno che rese dolci le acque salate del lago di Mara (Es I), 23-25), la verga con cui Mosè fece scaturire l'acqua nel deserto (Es 17, 5-7), l'asta attorno alla quale avvolse il serpente di rame ( $\mathrm{Nm} 2 \mathrm{2}, 8-9)$, l'arpa su cui Davide componeva i suoi salmi. Il rispecchiarsi delle profezie dell'Antico Testamento nel messaggio soterico del Vangelo si spinge fino a intravedere affinità che conferiscono significato alla lettera della storia sacra, senza dover ricorrere al sourasenso: come l'uomo è caduto nel peccato aprendo le braccia per cogliere il frutto proibito, cosi la restaurazione della sua purezza originaria avviene attraverso la croce su cui Cristo sta appeso con le braccia aperte, che è anche la postura dell'orante. Dunque Cristo prega in croce il Padre per la salvezza dell'uomo. ${ }^{45}$ Per questo materiale Grazzini avrà attinto a un repertorio, che era a disposizione un po' di tutti, data l'importanza che la predicazione aveva nella vita sociale (se egli non aveva qui presente una o più fonti precise), e con il quale egli ostenta una certa dimestichezza anche in contesti di tutt'altro genere. ${ }^{46}$ Su questo terreno abbastanza orientato, ma non problematico sul piano dottrinale, si innestano in maniera poco eclatante, e tuttavia di rilievo, proposizioni evangeliche in materia di redenzione. Una prima volta quando Grazzini cita la Prima lettera di Pietro I, I8-I9: 
per mezzo della Croce fu purgata la colpa de' miseri mortali, in quella guisa che dice san Pietro: «Non siete salvati né con oro, né con argento, ma con il prezioso Sangue di Giesù Cristo » sparso sopra del felice letto della Croce $;^{47}$

poi, nella chiusa, dove il valore salvifico della croce è ribadito senza citare il ruolo mediatore della Chiesa, e il cuore del fedele è chiamato ad accogliere direttamente la salvezza che promana dal crocifisso:

Questa [la croce] è quella, o cari Fratelli, e Padri, nella quale ognor riposta è nostra salute ${ }^{48}$

Or ricorriamo adunque a questo Santo Legno, il quale è stato sostenimento del nostro Salvatore, il quale ne doni sempre il mezzo a unirsi col Padre Eterno; nessuno si sdegni a quello mandare ora il suo cuore. ${ }^{49}$

La più significativa delle Orazioni è senza dubbio la seconda, anche la più lunga e la più efficace per chiarezza espositiva, nella quale ricorrono punti centrali delle dottrine evangeliche formulati con un linguaggio diretto e privo di sfumature. Il termine chiave "beneficio 》 ricorre cinque volte (altre due nella quarta orazione), ma la tesi dell'inutilità delle opere per la salvezza e della gratuità del dono della giustificazione, introdotta con la citazione di Paolo (Eb 3, I4-5), trova un'esposizione di ineccepibile chiarezza nelle seguentiparole:

E che noi tutti fussimo, e siamo per il peccato infermi, incarcerati, e strani, e del tutto morti, quanto al bene operare, e quanto a Dio, non credo, che alcuno capace di ragione ne dubiti, o dubitar ne possa. È venuto adunque Cristo, sanità, libertà, vita, e giustificazione nostra, è venuto per noi gratamente, non per alcuna opera di giustizia, come dice Paulo, che da noi fatta l'avesse a muovere a ciò fare, ma per sua misericordia e' ci ha fatto salvi per il lavacro del Sangue suo. Quanto manco era tenuto a venire, quanto più avevamo noi bisogno, che Cristo venisse (non potendo da noi rilevarci; imperocché chi è morto, è privo d'ogni opera, solo gli è data la imperfezione del corrompersi, e disfarsi del tutto) tanto è maggiore questo benefizio di tutti gli altri. ${ }^{50}$

Allo stesso modo è esposta in termini corretti la dottrina della giustizia imputata a proposito della salvezza dal diluvio concessa agli eletti nell'arca, che, come si è detto pocanzi, è allegoria della liberazione dal peccato attraverso il martirio di Cristo sulla croce. La grazia viene concessa al giusto non in quanto egli sia effettivamente giusto, condizione impossibile da raggiungere per l'uomo con i suoi mezzi, ma nell'unica forma in cui egli può attingervi, cioè attraverso l'imputazione della giustizia di Cristo: la colpa non gli viene imputata e perciò egli diviene giusto giudizialmente, pur rimanendo nella realtà peccatore: 
Le acque del Diluvio ricopersero la faccia di tutta la terra uccidendo ogni uomo, fuori che li pochi, che nell'Arca si salvarono, perché erano giusti, non però sì, che di natura loro non fussero peccatori, ma fatti giusti dalla liberalità e grazia di Dio, mediante la quale si giustifica l'empio. ${ }^{5 \mathrm{I}}$

L'esortazione a seguire l'esempio di Cristo per ottenere la salvezza è formulata con accenti che ricordano il capitolo $v$ del Beneficio "Come il cristiano si veste di Cristo », compreso la citazione da Lc 9, 23, che compare in entrambi gli scritti:

dalla pena di quello misurando la pena nostra, e dal gastigo dato in lui conoscendo quanto Iddio abbia in odio il peccato, e trovandocene pieni, ci verremo a confondere, a conoscerci, e in breve a disperarci di noi stessi; e questo è quello, che Cristo volendoci insegnare la via d'andare al Cielo ci insegnava dicendo: « Chi vuol venire doppo di me, annieghi se medesimo, e tolga la Croce sua, e seguitemi». Questa Croce della considerazione di noi stessi doviamo porci in su le spalle, la quale invero so, che ci parrà così grave, perché infatti ell'è così [...]. Rinunziato adunque, e diffidati di noi medesimi in tutto, seguitiamo in questa santa Passione questo Cristo crocifisso, e qui troveremo ogni contento, e ogni allegrezza nostra. ${ }^{2}$

Netto è anche il distacco dalla devozione tradizionale che insiste sugli aspetti patetici della Passione, con il risultato di colpire l'emotività dei fedeli, allontanandoli dal dono spirituale che è insito in essa e su cui si deve concentrare l'adorazione del crocefisso. E questo un tema centrale della predicazione ochiniana e della polemistica riformata, ad esempio di un Pietro Paolo Vergerio, intesa a confutare le componenti più irrazionali e arbitrarie del culto, le pratiche degenerate della liturgia cattolica e la manipolazione fratesca dell'ingenuità popolare. La prosa grazziniana è efficace e incisiva:

Né voglio io, che noi consideriamo questa Passione del Figliuol di Dio, come fanno certi, i quali mentreché raccontano una lunga istoria piena di varij, e miserandi casi, lasciando a dietro il frutto, e la considerazione migliore, o fanno una invettiva contro a Giuda, e contro alli Giudei autori di tanta scelleraggine in quanto a loro, che per far ciò la commessono; ma quanto alli fedeli, ministri di Dio in condurre a perfezione l'ordine della salute nostra; o sì a guisa di quelle donnicciuole riprese da Cristo gli vanno piangendo inutilmente dietro, come si farebbe, e si fa talora quando si sente, che uno innocente è stato morto. Non deve, Padri, e Fratelli in Cristo, tale essere la Cristiana contemplazione di questa santa passione; non contro alli crocifissori Ebrei incrudelire si debbe, perciò che essi furono, ancora che perciò non lo facessero, furono, dico, ministri a eseguire la volontà di Dio: contro alli nostri peccati veri, e soli crocefissori di Cristo doviamo incrudelire, contro di loro accenderci per ciò di tale odio, che a guisa di 
mortali nimici sempre gli abbiamo in dispetto, né sopra di Cristo come innocente morto pianger doviamo; imperocché egli giustamente morì, avendo preso sopra di sé il peccato nostro. ${ }^{53}$

Lasciato da parte il compianto lacrimoso del crocifisso, il fedele deve volgere il culto di esso in contemplazione gioiosa, perché rappresenta il dono volontario da parte di Dio della salvezza, attraverso l'espiazione sostitutiva nella persona del figlio. In consonanza con le tesi evangeliche, nel mistero della croce Grazzini vede il ripristino dello stato di perfezione e di comunione con Dio anteriore al peccato originale, senza che vi abbia parte la cooperazione umana. Proposta in questa luce, l'adorazione della croce diviene la commemorazione vivificatrice del dono spirituale della vita eterna elargito da Dio agli nomini. Di nuovo Paolo (I Cor 3, 2) sigilla questo accostamento gioioso al mistero della salvezza:

Lasciate adunque la storia della Passione da parte, e le invettive contro alli crocefissori, e il piangere delle donnicciuole, e così, come dice Paulo, poste giù le cose da parvoli piglieremo il cibo solido, e fermo, del quale cercando in questo santo giorno, troveremo, che nessuna cosa è, la quale ci possa dar tanto dolore, né tanto sbigottirci, quanto il considerare cristianamente la Passione di Cristo, né alcuna cosa tanto può rallegrarci, e confortarci, quanto la Passione di Cristo. ${ }^{54}$

Tutta la seconda parte dell'orazione consiste di un'appassionata variazione sull'antitesi morte-rinascita spirituale imperniata sul mistero della croce, unica via aperta all'uomo per sconfiggere il peccato e la morte, e ritornare a colui che l'ha creato simile sé.

Emerge, dunque, consapevole dai Sermoni, anche se in presenza di scarti e cambi di registro, una concezione razionale della religione e l'esigenza di una fede che, aderendo al messaggio originario delle Scritture, si configuri come esperienza spirituale metastorica, prescindendo dalle manifestazioni esteriori e secolari. E tuttavia difficile pensare che si possa concedere un credito incondizionato a questa visione depurata e rarefatta del sentimento religioso, e che ad essa si possa ricondurre tutta l'esperienza di credente di Grazzini e l'intera sua produzione sacra. A una devozione popolare, legata alle credenze e ai riti collettivi di una società pervasa di religiosità ingenua ai limiti della superstizione, riportano ben sei sonetti contenenti l'invocazione affinché cessi il cattivo tempo. In verità con sostanziali differenze tra loro. In un primo gruppo di quattro vanno posti $i$ due, Se per colpe del popolo ingrato $e$ Come già nel deserto umilemente, ${ }^{55}$ che l'edizione Moüke certifica composti per impetrare la pioggia dalla Madonna dell'Impruneta nell'ottobre I530, ${ }^{56}$ il che ne farebbe i primi testi poetici datati di Grazzini, e due analoghi, questa volta per far terminare le piogge eccessive, trasmessi dal Magliabechiano senza note 
di sorta: Saria forse giammai questo il secondo, Con quella alma pietà dolce infinita. Va però osservato - come ha fatto Plaisance ${ }^{57}$ - che tutti e quattro $i$ sonetti sono indirizzati non alla Madonna ma a Dio, il che cancella il ruolo avvocatizio di Maria e avvicina queste poesie alla soteriologia ocbiniana delle Orazioni, dove non a caso la Vergine non è mai nominata. Proclamando il valore esclusivo della croce come origine della giustificazione, Ochino sottrae alla figura della madre di Cristo la funzione mediatrice che le assegna la tradizione medievale, per riservarle tra le creature umane uno stato di elezione, in virtù dell'essere stata designata da Dio per accogliere Cristo ed essere vissuta nella luce beatificante della sua predicazione fino all'estremo sacrificio. ${ }^{5}$ La stessa scelta, che fa Grazzini in questi sonetti di richiamarsi a celebri episodi biblici, istituisce un parallelo con il popolo eletto che trascende il fine e la natura paganeggiante della supplica, per enunciare l'universale condizione di difetto, in cui versa l'umanità peccatrice, e invocare la prospettiva di un soccorso che solo può provenire all'uomo dolente e smarrito dalla "dolce pietà ch'avanza ogni nostro peccato » di un Dio generoso e clemente, disposto a elargire la grazia senza l'azione cooperante dell'nomo, solo per i meriti acquisiti da Cristo. L'arca di Noè, motivo pressoché obbligato nei sonetti sull'alluvione, in quanto figura della salvazione dell'umanità dal peccato per l'amore di Dio, suggella questo stato di attesa fiduciosa dei doni celesti, e testimonia chiara in Grazzini la coscienza della continuità tra il messaggio salvifico dell'Antico e del Nuovo Testamento culminante nella croce come atto estremo e definitivo del cammino di salvezza dell'uomo attraverso la storia: ${ }^{59}$

Se per colpe del popolo ingrato ne i secoli passati si ritrova che senza nulla dar rugiada, o piova stette ben trecent'anni il Ciel serrato, ${ }^{6}$ misero dunque il popol battezzato, dove ogni vizio fa l'ultima prova. Ma quella, Signor mio, dolce ti muova pietà ch'avanza ogni nostro peccato.

E l'humil gregge tua, che vagabonda erra, d'ogni altro vota, e di miseria piena, soccorri tosto, che 'n te solo ha spene.

E apri il Cielo, e fa' con larga vena l'acqua venire a rinfrescar la Terra onde il frutto ne dia, che l'huom mantiene.

Come già nel deserto humilemente aspettavan la manna giù dal Cielo i santi Padri, hor collo stesso zelo s'aspetta l'acqua dall'humana gente. 
Però, Signor, con quello amore ardente, che ti fece pigliar terrestre velo, e patir fame, sete, caldo e gielo, risguarda il popol tuo benignamente,

il qual pentito, e pien d'aspro dolore divotamente a te chiede mercede, a te chiede perdon, se mai ti spiacque,

e tutto pien di speranza, e di fede ti prega ${ }^{61}$ homai che dal Ciel mandi fuore con abbondanza, e quetamente l'acque.

Saria forse giammai questo il secondo diluvio, Eterno Dio, quando ti piacque anticamente col furor dell'acque purgar l'infetto e scellerato Mondo?

Ma se dal mar de' peccati profondo giusta cagion di punirlo ti piacque, che fia hor dunque? E se tanto ti spiacque quel secol, certo men che 'l nostro immondo,

Signore, il sangue ancor del tuo gran Figlio non era sparso, ch'acquistò per noi grazia infinita nel divin consiglio.

Però risguarda con pietoso ciglio gli error nostri non già, ma i merti suoi, e scampa il Mondo da sì gran periglio.

Con quella alma pietà dolce infinita, ${ }^{62}$ anzi caldo, affocato, ardente amore, che già ti mosse, eterno alto Signore, a dar, morendo in Croce, ${ }^{63}$ a noi la vita, volgi or gli occhi alla gente sbigottita, d'affanni carca, e colma di dolore, che, dolente e pentita d'ogni errore, cerca piangendo la tua santa aita.

E non voler la pioggia spessa e folta crescer così che steril sia la Terra, né dall'acque coperta un'altra volta.

Esaudi il popol tuo, ch'humil s'atterra e colla mente chiede, a te rivolta pace horamai doppo sì lunga guerra. ${ }^{64}$

Due ulteriori sonetti furono composti (ancora stando all'edizione settecentesca) per scongiurare l'alluvione nel novembre $1547,{ }^{65}$ il che proverebbe la costanza di Grazzini nel tempo con questo tipo di poesia d'occasione, ma con un 
sostanziale cambiamento: pur nel persistere dell' impostazione poetica rigorosa, che nulla concede ai risvolti meno nobili della circostanza, al centro è ora la figura mediatrice della Vergine. Il che porta a mutare il solenne tono biblico dei sonetti or ora citati nel registro accorato della chiamata in soccorso, con l'accento messo, come di prammatica, sulla sollecitudine materna della figura della Vergine, cui spetta il ruolo non solo della madre di Cristo, bensi di specifica avvocata e protettrice di Firenze nel momento del «periglio ». Si assiste insomma alla significativa rinuncia alla prospettiva universalistica dell'intera cristianità, il «popol battezzato » della prima coppia di sonetti, a beneficio di una più angusta dimensione municipale, espressa dalla preghiera corale della cittadinanza per scongiurare la minaccia incombente degli elementi:

Ecco, Donna del Ciel, ch'humile, e lieta la gloriosa tua città del giglio ti chiama per aiuto, e per consiglio nelle miserie sue, che non han meta.

Già mille volte $\mathrm{o}$ più mossero a pièta le preci sue ${ }^{66}$ e 'l suo vicin periglio per te sua Madre il tuo pietoso Figlio, nella cui sol bontà spera e s'acqueta.

Dunque i suoi preghi, e gli aspri danni atroci, e la sua speme in te fiano hora indarno, lasciando lei, ch'è tua, così perire?

Volgi, volgi omai gli occhi al tuo ${ }^{67}$ bell'Arno e odi a te ben mille e mille voci gridar tutte piangendo, e così dire:

«Se mai dentro i superni santi chiostri nel tuo candido petto pietà pose miseria estrema dell'humane cose, increscati hor, Maria, dei danni nostri.

Tu vedi, ohimé, che, quasi feri mostri, van divorando l'acque perigliose la vita nostra, se già con pietose preci al tuo Figlio il nostro mal non mostri.

Deh pregal, poi che 'l Cielo, e gli elementi non sol creò per noi, ma il proprio sangue sparger non si sdegnò per nostro amore, che voglia omai del popol suo, che langue, pietate haver, fermando agli aspri venti e alle spesse piogge il rio furore».

Siamo dunque dinanzi a due approcci radicalmente diversi a una religiosità popolare, cui Grazzini era legato per ambiente ed estrazione culturale e che 
costituivano uno stimolo concreto alla produzione poetica: in un caso egli si allinea all'orientamento evangelico delle Orazioni, nell'altro ricalca gli stereotipi di una religiosità semplificata ed emotiva, cui gli spiriti riformati guardavano con riprovazione, considerandola una forma di superstizione e di ignoranza che nulla aveva a che fare con la vera fede. Viene dunque naturale chiedersi se esistano in Grazzini due maniere distinte di poetare su cose sacre e se ad esse corrispondano due fasi diverse della sua evoluzione. Ora, la biografia grazziniana, cosi avara di dati cui appigliarsi specie negli anni della giovinezza, non ci autorizza in alcun modo a parlare di crisi o conversione spirituale, magari legata all'impressione suscitata dalle prediche fiorentine dell'Ochino, e poi di un ritorno entro $i$ confini di una devozione tradizionale. Se torniamo al sonetto rinterzato del 1538 , parrebbe che le poesie circolanti sotto il nome della marchesa di Pescara fossero di parecchio anteriori: composti « ab antico» le dice Grazzini. Non so quanto si debba dare credito a questa dicbiaraz̧ione di vetustà, ma si fatica a pensare che sonetti come $i$ due fatti risalire al IS3I potessero passare per fattura di Vittoria Colonna. ${ }^{68}$ Se, per esercizio congetturale, vogliamo supporre che gli apocrifi circolanti nel is 38 fossero quelli del manoscritto II.IV.I o quelli ad essi più simili tra quelli contenuti nel Magliabechiano, l'ipotesi ha più del persuasivo, anche se una lettura attenta rivela la sostanziale alterità di Grazzini dalle movenze della lirica spirituale della Pescara. Nellimpossibilità di fissare uno spartiacque tra la prima e la seconda maniera della poesia religiosa del poeta fiorentino, posto che ne sia esistito uno di rilievo dirimente, non resta che assumere a discrimine la data del I547, che abbiamo indicato sopra come decisiva nelle vicende culturali del ducato mediceo, e all'altezza della quale gli ultimi due sonetti meteorologici attestano che il distacco di Grazzini dalle posizioni evangeliche si era consumato.

Per tentare di articolare meglio questa ipotesi è necessario tornare ai due testimoni principali delle rime spirituali grazziniane e procedere a un esame più approfondito. Nel Magliabechiano le poesie si presentano sen₹a un ordine preciso. Valore del tutto estrinseco ba la triplice numerazione progressiva in caratteri arabi che distingue $i$ sonetti in tre gruppi: Io componimenti da c. $202 r$ a c. 206v, 24 da c. $208 r$ a c. $219 r, 7$ da c. $230 r$ a c. 233r; le carte restanti ospitano le quattro ottave, non numerate (c. 207r-v), l'egloga sulla Passione di Cristo (cc. 220r-226v), la quarta Orazione alla croce (cc. 226v-229v). Alcuni incidenti nella scrittura o trascuratezze nella mise en page (il sonetto Ahi quanto è presto, e come a fuggir leve è presente due volte, a c. 2I2v e a c. 2I6r; a c. 2I3v Varchi, dunque nei lacci, e negli inganni ba il primo verso irrelato; la prima quartina di Poi che sempre tornar veggio di gielo, trascritto per intero a c. $217 v$, 
si legge anche a c. $216 \mathrm{v}$ dal fondo del foglio; titolo e prima riga dell' Orazione sono in calce a c. 226v, invece di occupare, come sarebbe logico, il recto della carta successiva) e le correzioni o varianti apposte su alcuni componimenti provano con ogni evidenza che ci troviamo dinanzi a una raccolta compilata dall'autore per suo uso e consumo, senza seguire un disegno meditato. Quanto alla data della stesura del codice, $i$ sonetti con destinatario, come si vedrà più avanti, consentono di collocarla abbastanza in avanti, almeno agli anni Cinquanta.

Altra cosa è la piccola silloge del codice II.IV.I, che è per l'appunto un florilegio, preceduto dalla sua brava dedica: "Sonetti spirituali del Lasca Humyd.mo Della Achademia Fiorentina degl'Humydi, Indiritti allo Stradino Humido", per il quale la scelta dei componimenti non fu casuale - a cominciare dalla misura di dodici - e la successione segue una logica studiata. Dinanzi agli interrogativi che si aprono sulla genesi della raccoltina$i$ sonetti furono composti in sincrono per la medesima occasione, o furono estrapolati tra quelli già composti per essere montati in un insieme unitario? l'occasione era quella di fornire uno specimen alla neonata Accademia o fu girata ad essa una creazione preesistente? - l'ipotesi per cui propendo è che la corona sia stata confezionata ad hoc per gli Umidi utilizzando poesie composte prima. Come data dell'operazione dovremo prendere il termine a quo della fondazione dell'Accademia, novembre I540, al quale rimanda pure l" Oratione exortatoria recitata per uno Romito nella compagnia della Cicilia di Fiesole, l'anno I540 ", copiata subito dopo nel codice, che sarà stata recitata per la festa della santa, il 22 novembre: dunque primizie accademiche, che Grazzini si sarà affrettato a mettere a disposizione per il costituendo patrimonio comune del neonato consesso letterario. ${ }^{69}$ Anni dopo le poesie confluirono in ordine sparso nel deposito del Magliabechiano senza tenere conto dell'ormai dimenticata edizione prodotta per gli Umidi, tant'è vero che se ne smarri un pezzo, il sonetto II della corona, mancante nel Magliabechiano. ${ }^{70}$

Dunque la raccolta del ms. II.IV.I rappresenta per così dire la quintessenza, il fior fiore più rappresentativo della prima maniera della rimeria sacra grazziniana, d'ispirazione evangelica, e richiede pertanto un esame approfondito dei contenuti e del criterio con cui fu costituita. Una parte dei sonetti, $i$ numeri III, IV, X, XI, è riconducibile in maniera diretta al rito dell' adorazione della croce del Venerdì santo: il rapporto con l'occasione liturgica è marcato in maniera inequivoca dall'avverbio "oggi »: III, I: "Hoggi c'ba 'l sole $i$ bei lucenti rai 》; IV, Io: " col qual hoggi 'l gran sol della Iustitia »; X, I4: « hoggi pentito a lui chiede perdono $» ; X I, 7:$ " pietoso bavendo hoggi versato fore $» ;{ }^{71} i$ restanti presentano un contenuto più generico. I temi su cui insistono $i$ singoli componimenti sono $i$ seguenti: 
I il P. si pente degli errori commessi in passato e rivolge l'anima a Dio, fiducioso della infinita pietà del Signore, che lo fa sperare nella salvezza;

II il P. invoca la clemenza divina, implorando che gli siano rimessi i peccati in virtù della pietà di Dio, che è sceso in terra per redimere gli uomini;

III nel giorno della morte di Cristo il P. esorta l'anima a volgersi alla via della vera salvezza disdegnando le tentazioni mondane;

IV il P. invita il peccatore ad adorare la croce, per mezzo della quale l'umanità è stata redenta dal sacrificio di Cristo, e a mostrare così di essere degno di salire al cielo;

v invocazione alla Vergine affinché soccorra l'anima involta nei vizi pregando Gesù che la tragga a sé in cielo, umile e desiderosa di unirsi a lui;

VI dopo aver condotta una vita priva di speranze immerso nell'errore amoroso, il P., pieno di ardente zelo, rivolge tutti i pensieri a Dio e prega che la sua anima si ricongiunga a lui;

VII il P. è preso da un pianto dirotto al pensiero della morte; tre cose lo sgomentano: non sapere quando e come sopravverrà, il dolore che essa suscita e soprattutto il non conoscere se il suo destino sarà la grazia o la dannazione eterna;

VIII il P. ha vinto finalmente le illecebre di Amore e rivolto i suoi pensieri alla vera virtù; solo chi vuole essere vinto soggiace alla tirannia d'Amore;

IX un nuovo desiderio anima il P., ormai dimentico degli errori terreni e volto a una meta più alta; invoca Dio affinché lo aiuti a superare gli ostacoli sulla strada del vero;

$\mathrm{x}$ il P. invoca la Vergine affinché preghi il Redentore per le piaghe della croce che plachi il suo sdegno e si volga benevolo ai peccatori, non guardando alle loro colpe, ma al loro pentimento;

XI chi non può provare dolore dinanzi al sacrificio di Cristo in croce? Il P. invita i fedeli ad adorare colui che giace morto per avere dato ai peccatori la vita eterna;

XII chi confida nel Signore non smarrisce mai la via. Il P., che un tempo viveva nella schiavitù d'Amore, spera di andare in cielo e rimpiange di non essersi affrancato prima dai lacci amorosi.

Quello che emerge è un'architettura d'insieme, che viene a configurare la raccoltina come un microcanzoniere confezionato ad hoc per un pubblico intendente di poesia e vicino per sentimenti religiosi alle posizioni spiritualiste dell'autore. Tema della corona è un itinerarium in Deum che si incardina sui motivi della contrizione e del rinnovamento spirituale del penitente, nella speranza della vita eterna. Si va dalla proemiale invocazione della pietà divina (I-II) al disdegno del mondo per volgersi a Dio, solo datore della salute eterna (III), all'adorazione della croce, unica fonte della grazia (IV); seguono l'invocazione della Vergine come mediatrice e avvocata 
presso Cristo (V), un nuovo pentimento e innalzamento dell'anima a Dio $(V I)$, lo sgomento dinanzi all'insondabilità del mistero della predestinazione (VII), una nuova dicbiarazione di fede e l'invocazione a Dio (VIII$I X)$, una seconda invocazione alla Vergine $(X)$, ancora l'adorazione della Croce $(X I)$ e la conclusiva dichiarazione di irrevocabile rinuncia alle tentazioni terrene (XII).

Ora, se si abbandona questo quadro d'insieme e si passa all'esame ravvicinato, la sensazione di unità che si avverte al primo approccio viene meno ed emerge una testura assai più variegata di temi e suggestioni, non tutti univocamente riconducibili alla matrice da cui muove il progetto nel suo insieme. La tesi evangelica della giustificazione sostitutiva si affaccia dietro al sonetto IV, dall'enunciato fortemente assertivo: ${ }^{72}$

È questo quel sacrato legno, e santo dove nostra salute prima nacque? È questo il legno, dove morto giacque Chi 'l Mondo liberò d'eterno pianto?

Qui lasciò 'l mio Signor l'esangue ammanto tornando vittorioso u' pria gli piacque calcar il Ciel sovra le terre, e l'acque, benché per noi s'humiliassi tanto.

Dunque quest'è quel glorioso legno col qual hoggi 'l gran sol della Iustitia ci aperse il bel celeste e santo Regno: su peccator, mostra adornando 'l segno di dolce duolo, e d'amara letitia, che per lui in Ciel salir sei fatto degno.

Prima le quartine strutturate in forma di interrogazioni retoriche, con la variatio sintattica tra la prima, binata, e la seconda risolta in un unico giro avvolgente; poi le terzine aperte dalla martellante allitterazione iniziale e concluse con la parenesi di tono imperioso che racchiude nel duplice ossimoro disposto in chiasmo del verso I3 il paradosso salvifico della croce. La collazione col testo tràdito dal Magliabechiano, di cui fornisco di seguito le varianti sostanziali, offre qualche spunto meritevole di attenzione:

v. 4: colui, che ne scampò d'eterno pianto?

v. 5: Qui lasciò rotto il suo terrestre Manto

v. 8: chi per innalzar noi, s'abbassò tanto

v. 9: Questo è quel dunque fra i più cari legni $>$ Questo è quel sol tra i più pregiati legni

v. I I: il fio pagò dell'empie colpe nostre

v. I 2: ciascun dunque adorando 'l segno mostre. 
A parte il timbro più concreto dello scampò del v. 4 e l'accento spostato dalla prospettiva della giustificazione paradisiaca al presente della colpa umana al v. II, il dato più interessante è al v. , l'esangue del ms. II.IV.I, che non è proprio un sostituto equipollente del terrestre del Magliabechiano. La metafora " terrestre manto " per il corpo di Cristo pone infatti l'accento sull'unione ipostatica delle due nature di Cristo, che è, naturalmente, un motivo tradizionale, ma che nelle correnti riformatrici - ad esempio nel valdesianismo, in Calvino e in Zwingli - viene riletto spostando l'enfasi dalla prospettiva antropologica, in cui il credente si immedesima nel Cristo patiens e la salvezza scaturisce dalla mistica condivisione delle pene sofferte dal Salvatore, alla convinzione che il supplizio della croce abbia un valore salvifico in virtù della natura di chi lo ha patito. ${ }^{73}$ L'umanità del Redentore e l'enormità della sofferenza che egli ha accettato volontariamente per salvarci è uno dei punti su cui Grazzini si sofferma con maggiore energia nella seconda Orazione, citando il racconto evangelico della morte di Gesù di $\mathrm{Mt}$ 27, 46 e Mc I5, 34:

Cristo avendo fatto un fascio di tutti li peccati del mondo, e postili sopra le sue spalle, e andato con essi avanti al Padre Eterno per offerirli, Iddio, piamente parlando, veggendolo con tale peso addosso, e fatto protettore di noi altri miseri peccatori, l'ha avuto così in odio, se gli è così crucciato contro, gli ha dato così gran flagello, e tanta pena, che gli è stato forzato gridare in Croce: « Dio mio, Dio mio, perché mi hai abbandonato?». O grandezza del peccato! O miseria nostra grande! Se Iddio ha abbandonato, se Dio ha gastigato così questo suo diletto Cristo, il quale non aveva peccato, ma solo preso sopra di sé li nostri; se Dio ha guardato con tanta rigidezza questo suo Cristo, che portava avanti a lui questi nostri peccati $[\ldots]$ or che farà egli a noi miseri, e sciaurati peccatori ${ }^{74}$

Cristo sulla croce è stato abbandonato da Dio affinché patisse l'espiazione in quanto nomo, cioè sulla natura umana del Salvatore si esercitasse la giusta ira e la punizione di Dio contro l'immensità dei peccati dell'uomo, che da sé non riuscirebbe a giustificarsi. "Esangue » al posto di «terrestre » rimuove il riferimento all'umanità di Cristo, optando per una sottolineatura dell'intensità del supplizio, che attenua le implicazioni teologiche e insiste, pur sempre nella controllata cornice della metafora, sullo strazio del corpo del Redentore. E infatti l'aggettivo si ritrova puntualmente laddove lo spunto devozionale non si cristallizza nelle forme essenziali e imperiose del sonetto IV, e imbocca piuttosto la via della sollecitazione emotiva, ricorrendo al registro orroroso, in cui alla centralità teologica del "chi" muore sulla croce s'intreccia, senza distinguersi nettamente, la sollecitazione mistica del "come" muore. Cosi avviene nel sonetto XI, incentrato sulla contemplazione del corpo martoriato di Cristo, che sin dallincipit retorico si distingue per manifesta ricerca di pathos: 
Qual di rabbiosa tigre, o di crud'angue più invelenito, o più feroce cuore potrà mirando non sentir dolore, l'alto Figliuol di Dio ch'in croce langue, pallido, afflitto, macilente, esangue, e sol per nostro ben, per nostr'amore, pietoso havendo hoggi versato fore per cinque aspre ferite, tutto 'l sangue?

Alzate, peccator, gl'occhi, e 'l cortese Signor mirate, che cotanto v'ama, che per donarvi vita morto giace.

Udite l'alta voce che vi chiama a penitentia, e co' le braccia stese v'aspetta sol per dar l'eterna pace.

Non si tratta tuttavia soltanto di isolare $i$ due distinti registri impiegati da Grazzini nell'affrontare il tema della salvezza dell'umanità nella croce, motivo generatore e fulcro della raccoltina del ms. II.IV.I. Piuttosto, il dato più rilevante è che questo tema viene accostato da Grazzini eludendo un punto di vista propriamente teologico. Ė il sonetto proemiale a dare il vero timbro alla corona, accampando in limine, in forma nettissima, la mozione di un petrarchismo saldamente attestato, ben oltre il mero fatto linguistico, sulla ripresa del tema stoico della renovatio e del congedo dalla fallacia del mondo per aprirsi ai beni della vita spirituale:

Hor che da gl'occhi miei squarciat'è 'l velo, e rimpennato all'intelletto i vanni, che 'l Mondo scorgo, e i fallaci suoi inganni, non più le colpe mie nascondo, e celo; e veggio ben, sendone chiuso il Cielo, l'inferno aperto a miei perpetui danni, poscia che doppo, ahymé, tanti e tanti anni non muto viver bench'io cangi 'l pelo.

Ma perché la pietate alma infinita del nostro dolce eterno Redentore sempre tornar ci aspetta a miglior vita, pentito volgo a quella strada il core, la qual destri poggiar al Ciel n'aita, che bel fin fa chi in Dio ben vive, e more.

L'apertura è una eclatante dichiarazione di fedeltà al modello, che la seconda quartina rafforza accampando l'antitesi Cielo/inferno e un trasparente stilema petrarchesco quale "non muto viver bench'io cangi'l pelo $»^{75}-$ cosi 
caratteristico da essere riproposto nel son. VI, 3-4: "l'alma vagar nella prigion d'Amore / dov'ho i costumi variati, e'lpelo » (e poi, come si vedrà, nei sonetti con destinatario) -, prima che nelle terzine il proposito penitenziale si concretizzi nel progetto di «poggiare al cielo » vivendo e morendo in Dio. A questa mozione intimista e psicologica, su cui s'innesta l'adorazione della croce, non sarà da concedere una patente di autobiografismo - anzi, al contrario, è questo, il coté più letterario delle poesie-, ma proprio perciò esso costituisce la struttura portante, il codice poetico su cui poggiano le fondamenta dell'esperimento tentato. Laddove esprime il congedo dell'anima dalle cure terrene per innalzarsi alla vita dello spirito e all'unione con Dio, Grazzini esibisce un'orchestrazione saldamente ancorata ai grandi movimenti strutturanti del Canzoniere e concepisce tale processo innanzi tutto come percorso penitenziale, in cui il peccatore è chiamato ad imprimere alla propria esistenza una svolta che lo conduca con animo rinnovato e sincero al mistero salvifico della croce e al dono della grazia. Non è un caso che questo cammino di indiamento sia espresso attraverso la metafora del viaggio, onusta di precedenti romanzi e umanistici per significare itinerari di perfezionamento etico e spirituale, ma sostanziale sottoesposizione dell'iperbole dell'anima verso Dio che aleggia negli scritti degli animi più ispirati della Riforma, specie se, come avviene nell'esemplare sotto questo punto di vista sonetto IX, scoperti materiali del Canzoniere concorrono a colorire l'incertezza e la vulnerabilità, cui è esposto il poeta nei termini propri dell'accidia petrarchesca:

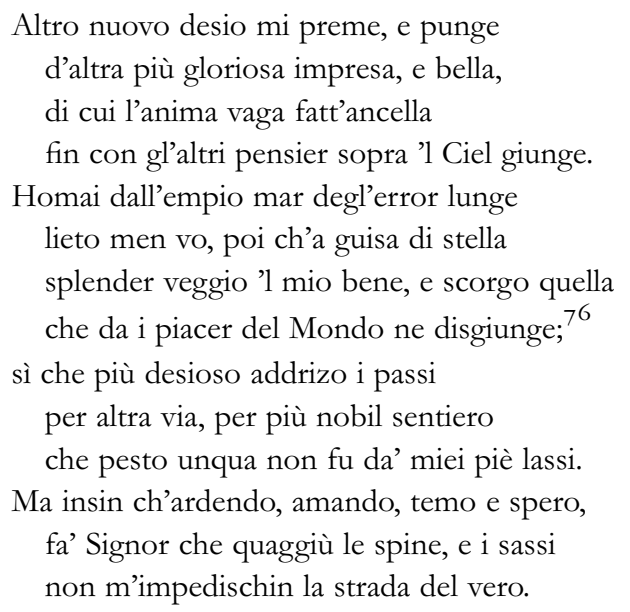

$\dot{E}$, in definitiva, l'adesione allo psicologismo petrarchesco, il veicolo per riportare a una dimensione più consentanea le istanze teologiche abbracciate dal poeta, attraverso lo schema di una biografia morale, cosi come il modello del Canzoniere autorevolmente offriva a chi nel Cinquecento si proponesse di 
poetare sul mondo interiore dell'uomo. Ciò non significa che il risultato sia sempre intenso e profondo. Anzi, piuttosto il contrario. L'armamentario formale dispiegato nei sonetti - tendenza alla struttura bipartita delle poesie e dei singoli versi, rinuncia all'inarcatura sintattica, uniformità nella costruzione dell'endecasillabo, propensione alla dittologia, alla ripresa, all'elenco palesa un'osservanza petrarchista insistente sui tratti più genuini e originari del modello, lontana dalla rigenerazione del codice impressa dalla linea tragica del petrarchismo cinquecentesco, che ha il suo archetipo nel canzoniere dellacasiano e impronta di sé la lirica della seconda metà del secolo, ma anche lontana dalle istanze intellettuali del platonismo che il modello petrarchesco viene ad ospitare nella rilettura bembiana, modello principe per la lirica più impegnata dei decenni precedenti, a cominciare proprio da Vittoria Colonna. Cade a proposito un'osservazione di Mila Mazzetti, in un penetrante saggio del 1973 sulle rime della Pescara, che applicata e contrario a Grazzini aiuta a cogliere la sua distanza dagli interpreti più avvertiti della temperie religiosa contemporanea e la strutturale deficienza della sua poesia sacra a rielaborare il modello trecentesco in una nuova forma spirituale adeguata ai tempi: "la Colonna - scrive Mazzetti - si allontana totalmente, per importanza e significato, dal momento spirituale della poesia del Petrarchismo presente in altri canzonieri come ripetizione del pellegrinaggio spirituale del Petrarca, quello del ravvedimento e della confessione $\gg{ }^{77}$ Nelle rime sacre grazziniane la rigenerazione spirituale del credente si attua nei termini di un itinerario espiatorio e di perfezionamento coronato dalla grazia, ma è difficile avvertire quell'autentico scatto in avanti, quell'anelito verso una dimensione nuova che trascenda l'esistenza storica dell'uomo, che è il tratto portante delle rime della Colonna e la sua dimensione profetica. Al contrario, l'avvertimento in Grazzini dello stato d'imperfezione, in cui il peccato dei progenitori ha precipitato l'uomo, senza che egli possa emendarsi con le sue sole forze - da cui l'implorazione sottomessa del Salvatore e la figura mediatrice della Vergine -, tende a fissarsi nella rappresentazione concreta di tale manchevolezza, invece di attenersi al livello astratto dell'avvertimento di una colpa primigenia come intima insufficienza della condizione umana che mina alla base l'atto della volontà. Grazzini interpreta questo stato universale dell'umanità immersa nel male per ontologia materializzandolo nella colpa amorosa, che costituisce una lettura alquanto riduttiva della dialettica tra aspirazione al divino e passioni terrene, ma soprattutto tale stato si colloca in obiettiva estraneità alla teoria platonizzante dell'amore espressa in caposaldi del classicismo cinquecentesco come gli Asolani o il IV libro del Cortegiano e che aveva rappresentato il più sofisticato prodotto della cultura rinascimentale fino a quel momento. Per Grazzini la passione amorosa rappresenta una condizione impediente per il percorso verso la grazia, da cui prendere le distanze in 
termini di un inconciliabile aut-aut. Esemplare a questo proposito l'attacco del sonetto VIII:

$$
\begin{aligned}
& \text { Già mai non credett'io da gl'empi, e feri } \\
& \text { gioghi d'Amor ritrar l'anima ardente, } \\
& \text { e hor la sento libera, e possente } \\
& \text { gl'amorosi schernir falsi piaceri. }
\end{aligned}
$$

Ma il tema amoroso, nei termini icastici di servitù d'Amore, s'insinua anche nel sonetto finale, che con una certa goffaggine proclama l'avvenuta conversione del peccatore pentito, e il rammarico di non essersi liberato prima dalle panie amorose. Peraltro, tornando per un momento al piano teologico, il sonetto enuncia la dottrina della salvezza in una forma assai diversa dalla tesi dell'espiazione sostitutiva, che abbiamo visto espressa in maniera molto consapevole nella seconda Orazione e adombrata in alcuni dei sonetti. Ora al centro non è più il tema della giustizia imputata, ciò̀ il beneficio gratuito elargito da Dio ai predestinati nonostante persista in loro la condizione di peccatori, ma si intravede, nei termini della dottrina cattolica, la possibilità di una giustificazione reale e del recupero dello stato di purezza originaria attraverso il pentimento e la rigenerazione spirituale del credente, dunque si restituisce all'nomo la possibilità di una collaborazione alla concessione della grazia, che le tesi evangeliche escludevano. Cosi riacquista tutto il suo peso il culto della Vergine, come figura intercedente necessaria alla mitigazione della giusta ira divina e alla copiosa e rapida elargizione della grazia agli nomini, che altrimenti tarderebbe a causa delle loro colpe. Costruiti sul medesimo schema - l'invocazione nella prima quartina e la richiesta di intercessione a partire dal verso seguente: $V$, s: "prega'l tuo Figlio, la cui man pietosa », X, s: "prega'l tuo Figlio, e nostro Redentore »-, i due sonetti mariani della corona si distinguono per $i$ diversi accenti con cui la figura soccorritrice della Madonna viene chiamata in causa; come figura umanamente adiutrice nel sonetto $\mathrm{V}$ :

$$
\begin{gathered}
\text { Madre del vero Dio, figliuola, e sposa, } \\
\text { deh Vergin santa, le mie preci ascolta, } \\
\text { soccorri all'alma, che ne' vitii involta } \\
\text { per questa valle errando va dubbiosa; }
\end{gathered}
$$

come celeste cooperatrice della grazia divina nel sonetto $x$ :

$$
\begin{aligned}
& \text { Pura luce infinita, almo splendore, } \\
& \text { ch'adorni il Cielo, e 'l Mondo fai beato, } \\
& \text { Vergine bella, il cui valor pregiato } \\
& \text { fé la gratia di Dio venir maggiore. }{ }^{79}
\end{aligned}
$$


La cooptazione della Madonna accanto a Dio e a Cristo nella Passione in termini che valicano la mera attanzialità si palesa in maniera ancora più netta in un sonetto del Magliabechiano esterno alla corona del ms. II.IV.I:

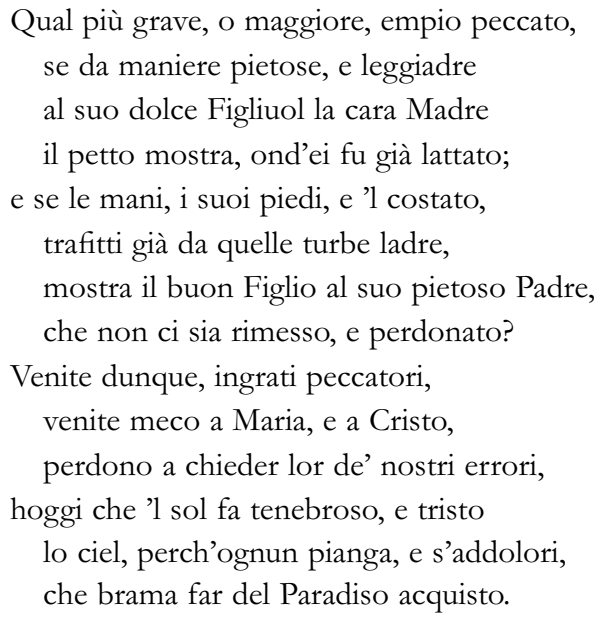

In perfetta simmetria con il sonetto proemiale, il percorso salvifico inscenato nella corona si chiude nel sonetto XII in un clima penitenziale, con il proposito del poeta, abbandonate le cure del mondo e intrapresa la retta via, di proseguire nella nuova vita, nella speranza di accedere un giorno alla beatitudine:

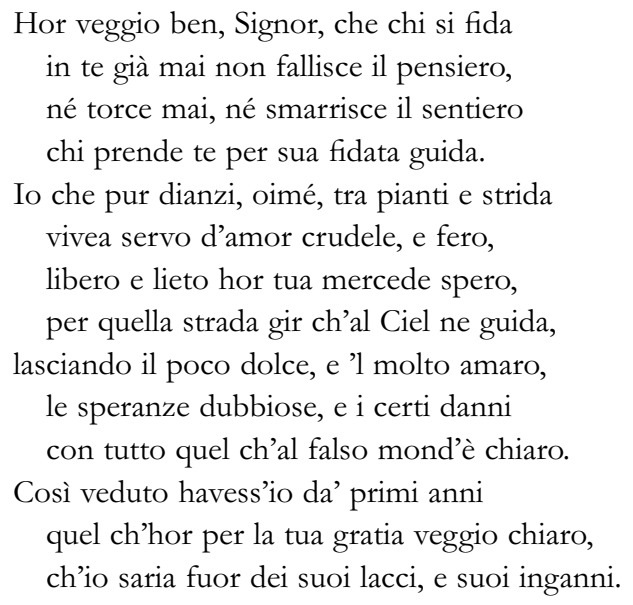

All'opposto, il sonetto VII aveva toccato il punto nevralgico della predestinazione per dare voce con toni laceranti allo sgomento in cui essa getta il cuore del credente: 


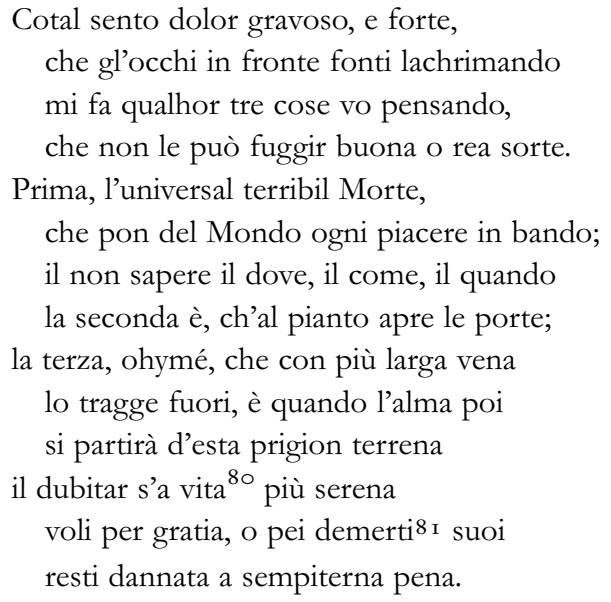

Lo smarrimento che esprimono questi versi permette di misurare ancora la distanza che li separa dalle poesie della marchesa di Pescara. In Dio la Colonna non vede il Dio giudice dell'Antico Testamento, ma il Dio padre ed elargitore del perdono, che ha compreso l'errore degli nomini e lo ha espiato nella persona di Cristo crocefisso; cosicché Vittoria aspira a sottomettersi al giudizio divino, certa della misericordia del Signore, ed esprime con toni di devozione anelante l'attesa della morte come momento dell'agognato ricongiungimento con il Padreterno e inizio della vera vita, cioè congedo dal mondo in un'autentica metanoia che innalza l'uomo alla sua superiore essenza. In Grazzini, piuttosto, il credente è posto dinanzi all'evento culminante della fede cristiana in uno stato di soggezione e di passività devota. Egli interpreta l'argomento dei sonetti come una volonterosa dichiarazione di fede, meglio che come un dischiudere la propria anima di fronte al mistero ineffabile di Dio. Nelle sue poesie la spiritualità consiste in pentimento e rigenerazione morale, ma il ricongiungimento con l'assoluto resta una prospettiva remota, cui tendere come a un evento che non è in grado di sconvolgere con la sua imminenza l'animo umano. La condizione di colpa in cui l'uomo si trova immerso in virtù del peccato adamitico e la giustificazione, che nelle anime più profonde $e$ sensibili dell'evangelismo italiano suscitarono vertiginose prospettive di esame interiore, si irrigidiscono in un contrasto tra aspirazione alla salvezza, per lo meno chiaramente intravista e invocata, e strutturale insufficienza del credente, risolto, con sostanziale ambiguità, ora con l'adesione alla tesi riformata della giustizia sostitutiva, ora con l'avvicinamento alla teoria cattolica della cooperazione umana alla grazia.

Il disimpegno dalle tematiche teologiche e l'imporsi di un'impostazione penitenziale è la chiave che ci permette di penetrare anche dentro $i$ sonetti tramandatici dal solo Magliabechiano, per $i$ quali è da ipotizzare una datazione 
posteriore alla corona del ms. II.IV.I, con qualche opportunità di collocarli meglio negli anni solo per quelli con destinatario. Appena un breve accenno dedicheremo all'egloga sulla Passione e morte del figliuol di Dio, per la quale è verosimile la data del I566, essendo l'ultima delle dieci che furono presentate in quell' anno ai censori all'Accademia Fiorentina per la riammissione. Più che « un po' troppo descrittiva », come la giudica Plaisance, ${ }^{82}$ la direi sostanzialmente fallita nel tentativo, sperimentato ad altri livelli dal Sannazaro del De partu Virginis, di convogliare nel registro pastorale contenuti religiosi, ormai non solo rinunciatari sul piano dellimpegno dottrinale, ma anche privi di autentiche vibrazioni emotive, sicché il risultato è quello di un esercizio conciliato e aproblematico, adatto allo scopo che si prefiggeva.

Sciolti da implicazioni dottrinali, $i$ sonetti con destinatario riducono l'ispirazione religiosa a pensosa meditaz̧ione sulla caducità della vita e sulla fede come unico esito di salvezza, commiato dai valori mondani e conversione ai beni dello spirito, a condizione però che non si pensi a ciò come a un itinerario intellettuale verso Dio. Il codice petrarchesco, dominante in maniera imperiosa, tende a polarizzare il contenuto delle poesie in una dicotomia che Grazzini modula con una serie di variazioni sul tema, insistendo sul motivo della fralezza della vita terrena e del grave pondo che impedisce di innalzarsi alle gioie celesti. Si vedano alcuni casi esemplari:

Questi occhi, e questi piedi, che mi fanno veder per tutto, e gire ove a me pare, coll'altre membra all'uom sì dolci e care, cenere tosto, e polvere saranno.

Così le glorie umane a terra vanno, né si può lor riparo o schermo fare; sì fugge il tempo senza mai tornare, con nostro immenso, e sempiterno danno.

Ohimé, stamane era io giovine, e forte (o vita nostra transitoria, e breve!), oggi son veglio, e frale, e presso a morte.

O Mondo rio, da te non si riceve se non oltraggio! Ahi nostra dura sorte, ch'altro siam noi, ch'al sol falda di neve?

Ahi quanto è presto, e come a fuggir leve questo giorno mortal, che vita ha nome! Dianzi le rose, hor il ghiaccio, e la neve; hier brune, oggi son bianche queste chiome.

Ond'io, Signor, per cui grazia riceve l'human legnaggio, a te ricorro, come a suo buon Padre ingrato figlio deve, 
ch'ha dispregiato, e schernito il suo nome,

e prego te, somma bontà infinita,

che di quei folli errori, ond'io vaneggio,

prender non vogli, ohimé, giusta vendetta.

Ma dammi, Padre Eterno, spazio e vita

da potermi pentir, poscia ch'io veggio

sparire il tempo via con tanta fretta.

Hor che dal Mondo, e dal suo cieco honore

mi parto sazio, e drizzo al Ciel la mente,

hor ch'arder l'alma mia tutta si sente

del tuo sagrato accesa, e santo amore,

piangendo, e sospirando a te, Signore

e Re del Ciel, m'inchino humilemente,

e dei miei falli pentito, e dolente

mercé chieggio, e perdon con tutto il core,

e reverente prego che ne dia

lume cotal, che tra queste ne scorga

tenebre folte la diritta via.

Deh la tua santa man cotanto porga

aiuto alla bramosa voglia mia,

che dal fango del Mondo al Ciel risorga.

All'ultimo verso del terzo sonetto accosteremo " la tragga fuor del fango, ov'è sepolta ", detto dell'anima nel son. V, 6 secondo la lezione del Magliabechiano - alternativo al meno icastico "la tragga de i peccati ov'è sepolta» del ms. II.IV.I - a testimonianza del ricorso alle tinte forti laddove Grazzini si impegna a tratteggiare il contrasto Cielo/Virtu-Terra/Vizio e della degradazione che caratterizag il polo inferiore, come evidenzia anche un'altra lezione alternativa del Magliabechiano, son. III, II: "lunge dal Mondo rio, che si n'adombra ", che traduce in espressa malvagità la più blanda condanna di fallacia della redazione del ms. II.IV.I: " lunge dal Mondo van che si n'adombra ». E con ciò chiudo la campionatura sulle varianti tra $i$ due testimoni con il giudizio, provvisorio in vista di un'auspicabile edizione critica, di una maggiore asprezza del testo del Magliabechiano, rispetto alle scelte piu sfumate dell'altro testimone, il che autorizza a pensare a un supplemento di labor limae per $i$ sonetti destinati alla pubblicazione, che abbia ripulito il lessico da espressioni estranee alle istanze di un petrarchismo più selettivo, sensibile all'essenzializzazione del linguaggio.

Anche nei sonetti con destinatario la condizione di difetto, in cui si dibatte il poeta, prende le fattezze della passione amorosa che si insignorisce dell'animo, sicché l'invocazione ansiosa a Dio si concreta in un'istanza di affrancamento dalla più cieca e vana delle tentazioni terrene per rivolgersi alle imperiture gioie 
celesti, senza però che questo anelito si articoli in una proposta definita sul piano teologico:

Tu vedi, Eterno Re, nella cui mano della Terra, e del Ciel pende il governo, in che doglioso, e periglioso inferno sepolto viva, ohimé, da te lontano.

Dammi, ond'io possa il desir cieco, e vano frenare affatto, ch'hor sì chiaro scerno, e questo orrido mio, rabbioso verno converti in dolce april, quieto, e piano.

Amor sopra di me s'è fatto donno, e mi sprona, e mi volge, e gira intorno come gli piace in dolorose tempre.

Scampane dunque tu, Signore addorno, poiché le forze mie da sé non ponno, ch'io sarò tuo fedel, vivendo, sempre.

La fuga dal «mare d'errore » del mondo è la cifra su cui s'imperniano con modalità seriale questi sonetti, nei quali gli altri spunti meditativi che si affiancano al motivo amoroso non coagulano in uno scavo esistenziale; al contrario, si ha la sensazione del costituirsi di una maniera, dove l'elevato livello formale del linguaggio non riesce a riscattare l'asfissia dell'ispirazione. Valga anche qui un esempio per intero e per il resto alcuni excerpta. Cosi il primo dei due sonetti a Lorenzo Scala che leggiamo nell'edizione Moücke (I, pp. 5-б):

A questo Mondo rio, che sé non tiene, all'empio disleal tiranno Amore volgendo il tergo, per la via d'onore drizzate i passi a più beata spene,

Scala gentile, a voi non s'appartiene spender, seguendo il volgo, i giorni e l'ore dubbioso sempre in questo mar d'errore, ch'è tutto pien di scogli e di sirene,

e ne' paesi strani al caldo e al gielo gir travagliando, e struggendo la vita, per un ben acquistar che poco dura.

Levate meco i pensier vostri al Cielo, dove ricchezza trovasi infinita, che 'l Tempo mai non guasta, e Morte fura.

La replica di Grazzini al sonetto responsivo dello Scala, Amor, Lasca gentil, da cui mi viene, ${ }^{8}$ svela quale sia l'ipotesto su cui stanno lavorando 
$i$ due: la canzone per eccellenza meditativa del Canzoniere: I' vo pensando, e nel pensier m'assale, di cui Grazzini esibisce a mo' di emblema la sentenza finale:

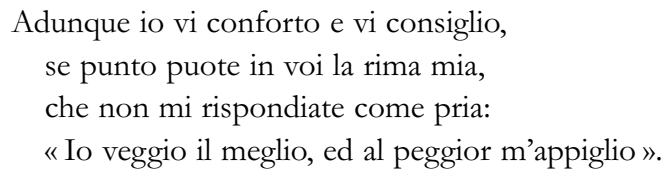

Ma il medesimo ipotesto si avverte anche nel sonetto a Giovambattista Cini, ${ }^{84}$ dove la lacerazione che attanaglia la coscienza del poeta è enunciata in toni dilemmatici e conclusa con la richiesta di dare su di essa "final sentenza 》 (v. I4), che costituisce l'invenzione su cui s'impernia la poesia:

S’io veggo certo, e conosco il mio bene perché non seguo, e non abbraccio il vero? S'io scorgo chiaro, e spedito il sentiero che guida dritto a Dio, chi ne ritiene?

Il sonetto a Guglielmo Martelli, Mirate, Martel mio, come repente, composto nel giorno del Venerdì santo (al v. II, Cristo "per darne vita in Croce oggi morio »), assume $i$ toni di un vero e proprio memento mori, espresso in modi piuttosto accesi mediante il ricorso all'ipotiposi, vv. 5-8:

Superba in vista ognor si vede, e sente

la Morte starci sopra, e già vi azzanna;

prendete prima la celeste manna, ché passa il vostro dì puro, e lucente.

Poi Grazzini non sa rinunciare a tradurre in concreto l'esortazione morale e nella seconda terzina specifica cosa sia che minacci la salvezza dell'anima del suo giovane interlocutore:

Voi siete hor qui, pensate alla partita, né vogliate ch'Amor fallace, e rio vi prive il corpo, e l'anima di vita.

Nel sonetto a Vincen₹o Alamanni Sendo voi nell'etade ancora acerba, per il quale dovremo arrestarci alla metà degli anni Cinquanta dato che l'Alamanni era nato il is gennaio I536, Grazzini si fa mentore dell'amico per indrizzarlo ai superiori valori della fede, vv. 9-I4:

Non ponete di qua vostro disio, ove la Morte, il Tempo, e la Fortuna fanno sempre di noi prede sicure; 
ma rivolgete i pensier tutti in una

veglia, e quella drizzate solo a Dio,

che tutte altre son basse, e vane cure.

Senza tema di peccare di dismisura e di fallire la soglia del grandioso, che in effetti non sembra alla portata di questi versi, in Volgete gli occhi, disiosi e 'ntenti Bastiano Antinori, nato nel I524, consolo dell'Accademia Fiorentina nel is 6s e tra i deputati per la correzione del Decameron per l'edizione del I573, viene invitato a contemplare le glorie dei grandi del passato spente dal tempo e a condividere con il poeta il contemptus mundi che ne consegue, vV. I2-I4:

Hor dunque voi, questo vil Mondo, e frale, che v'ha più volte schernito, e 'ngannato, lasciate meco, e 'l cor drizzate a Dio.

E singolare che gli unici sonetti, che abbandonano il registro penitenziale, incentrato sulla vocazione meramente ottativa del poeta e dei suoi sodali a Dio, sono dedicati a due donne, Laura Battiferri e la cortigiana redenta Giulia Napoletana. Non ci sono indizi testuali per interpretare queste due signore come figure della Vergine, ma non si può negare che alla costitutiva inettitudine del poeta e dei destinatari maschili ad attingere la meta della beatitudine si contrappone per le due donne una fulgida prospettiva di gioie celesti, anzi la possibilità di accostarvisi già in questa vita. La Battiferri, a Firenze dal I550 come sposa di Bartolomeo Ammannati, è presentata in Poi che tra le ricchezze, e glorie humane come una creatura angelica, "fuor d'ogni natural terren costume» (v. 2), dotata di "più ch'buman vivace lume 》 (v.3), la quale "volando al Ciel con sagre piume » $(v .7)$, lascia le ombre e séguita il vero, e di cui si può dire infine: "Dunque, o beata Voi, che già nel Mondo / cominciate a goder l'Eterno Bene » (vv. 13-I4). Il prevalere in questi versi di quella che si è ormai costituita in maniera, anche con la valenza mondana di tributo a figure in vista della società fiorentina del momento, non è tale tuttavia da trascendere il dato reale che origina questa poesia. Ciò si vede bene nei due sonetti alla cortigiana Giulia Napoletana, che Grazzini compose quando la donna lascio la vita dissoluta ed entrò nel monastero delle Convertite, ${ }^{85}$ evento salutato anche da Giovanni di Benedetto da Pistoia, col sonetto Anima bella, che dell'ombre uscita, ${ }^{86} e$ da Benedetto Varchi, col sonetto Hor che la bella Giulia vostra il crine, diretto a Giulio Scala, fratello di Loren $₹ 0 .{ }^{87}$ Posteriori a questa conversione devono essere le ottave Se ben voi sete de' cocchi private, composte da Grazzini sulla riforma degli Ufficiali dell'onestà che, tra le varie misure prese contro le femmine di mondo, prevedeva 
il divieto di usare mezzi di trasporto in città, salvo di notte e in orari concordati. Il poeta vi esorta le meretrici fiorentine a redimersi e ad entrare tra le Convertite, portando ad esempio la sfortunata sorte della "meschina» Giulia e della Diana fiorentina, già ricche e prospere, ora vecchie e inferme, particolare questo che posterga le ottave ai sonetti, dove Giulia è ritratta ancora avvenente, $e$ in ciò consiste appunto il valore della sua conversione. ${ }^{88}$ $\mathrm{Nei}$ due sonetti il consueto armamentario devoto e lo specifico corredo inventivo richiesto dal caso (l'inevitabile topos della Maddalena) non conseguono del tutto l'obiettivo di sublimare l'occasione biografica che li origina nello stereotipo espiatorio della peccatrice redenta e resa santa dal pentimento; al contrario, il finale del secondo sonetto lascia prosaicamente intravedere il pericolo di assalti del Maligno e il rischio di deflettere dalla strada intrapresa della salvezza. Eventualità che $i$ documenti coevi testimoniano come non eccezionale: il ravvedimento delle meretrici era talora transitorio o simulato per ragioni di calcolo, e non mancavano $i$ casi in cui le ricoverate ricadevano nella condotta dissoluta. L'effetto delle due poesie è perciò una singolare coabitazione di ascesi, che redime e cancella l'esperienza peccaminosa, e fragilità della carne quale persistente ostacolo sulla via della salvezza, che inficia in buona sostanza la prospettiva soterica e vi getta sopra un'ombra di pragmatico scetticismo:

Giulia, che 'l Mondo t'hai goduto, e hora, illuminato il petto, acceso il core da divin raggio, e da celeste ardore, il Paradiso godera'ti anchora, io, che le tue bellezze ad hora ad hora lodai cantando, e diedi eterno honore, che devrò far, se per la via migliore salir ti veggio a vicitar l'Aurora?

Donna invitta, e d'ogni lode piena, che conosciuto il rio Mondo fallace, che per un sol contento ha mille affanni, fatt'hai come già fé la Maddalena, che per trovare in Ciel l'eterna pace, cangiò modi, e costumi, e vita e panni.

Fabio, che 'n Ciel vederti ancor disia, dov'hor sì vive felice, e beato, l'Eterno Re per te tanto ha pregato che 'ntrata se' per la diritta via.

O Giulia, o Giulia non più bella e ria, ma bella e santa, il tuo cammin lodato segui pur lieta là 've t'ha chiamato 
il Figliuol glorioso di Maria.

Non ti volgere indietro, o da man manca

i passi gira, perch'agievolmente

errar potresti, e smarrir il sentiero.

E se talor la carne viene stanca, sia lo spirito pronto, e stieti a mente

in Dio porre, e fermare ogni pensiero.

Si avverte, al cospetto di questi versi torniti con calibrata eleganza il senso di sazietà che trasmette tanta rimeria d'occasione per battesimi, matrimoni, morti, monacazioni e via dicendo, presenza costante nella nostra letteratura a partire dalla seconda metà del XVI secolo fino all'Arcadia. Triste epilogo per uno dei rimatori burleschi più brillanti del secolo e seguace non ingenuo del verbo petrarchesco nella prima maniera delle sue rime spirituali.

Uno sguardo un po' più attento richiedono i sonetti spirituali che Grazzini scambiò con Benedetto Varchi, tramandatici dal Magliabechiano e, autografi, dal Banco Rari 59, parte della più ampia corrispondenza in versi intercorsa tra $i$ due letterati, per la quale, in attesa di una ricostruzione più affidabile, ci atteniamo alle edizioni cinquecentesche dei sonetti di Varchi $e$ all'edizione Moüke, parzialmente seguite dalla stampa ottocentesca dell'opera varchiana. Lo scambio poetico Grazzini-Varchi su temi spirituali dovrebbe dunque articolarsi in questi segmenti (l'ordine non costituisce una cronologia):

Grazzini: Come è, Varchi, di nuovo in voi risorto VARCHI: La fiamma ch'io portai nel core, e porto ${ }^{89}$

Grazzini: Tempo è homai, poi che cangiate il pelo VARCHI: Se bene io cangio d'ora in ora il pelo ${ }^{\circ}$

VARChI: Lasca, altro Febo, altra Minerva oma ${ }^{9 \mathrm{I}}$

Grazzini: Varchi, dunque nei lacci, e negli aguati

Grazzini: Temp'è, Varchi, hoggimai ch'affatto il core VArchi: Cosi se piace a lui, Lasca, il Signore ${ }^{22}$

Ora, che non ci si trovi dinanzi a componimenti vincolati ne varietur a un meccanismo corresponsivo concluso e ad personam, lo prova il fatto che Temp'è, Varchi, hoggimai ch'affatto il core, trasmesso con questo incipit dal Banco Rari 59, è presente nel Magliabechiano con il primo verso modificato in Temp'è bene hoggimai ch'affatto il core $e$ reintestato "Al Cavalier Fra Pagol del Rosso» su rasura della primitiva intitolazione "A Ms. Benedetto Varchi». L'adattamento risalirà a non prima del I 566: il Varchi era morto l'anno prima; il Del Rosso, già esule antimedi- 
ceo, riacquistò allora la libertà dopo la lunga carceraz̧ione inflittagli da Cosimo I nella torre di Pisa e fu riammesso nella vita culturale cittadina con l'ascrizione all'Accademia Fiorentina, poco prima di uscire definitivamente di scena il 13 gennaio I569, salutato da Grazzini con il sonetto Come siano, ohimé, fallaci, e torte. ${ }^{93}$ Il fatto che il componimento fosse smistato con disinvoltura su un altro destinatario non significa per forza che si debba negare ogni serietà al dialogo in versi imbastito dai due poeti. Entro lo schematismo condiviso di matrice petrarchesca, che tende a richiudere il confronto in uno schema precostituito, affiora nelle responsive del Varchi un dissenso autentico, che va a colpire proprio il nucleo forte dello spiritualismo grazziniano prima e seconda maniera, e cioè la sua natura penitenziale e ascetica, il rigetto in blocco delle attrattive del secolo e tra esse in primis dell'amore. Nelle due coppie di sonetti che propongo di seguito Varchi replica asciuttamente, con toni che non si possono dire formali, opponendo una concezione platonizzante dell' amore e l'accusa a Grazzini di essere zelatore di un'etica piu adatta alla creduloneria popolare che a un intellettuale par suo:

Come è, Varchi, di nuovo in voi risorto foco amoroso, che v'incenda il core? Dunque è acceso, e vivo quell'ardore, in voi già tanto tempo spento, e morto?

Io pur credea che già vi fuste accorto a mille prove che chi serve Amore prova, meschin, con suo danno e disnore, lungo e gran duol, per piacer poco e corto.

Mirate il gran periglio a cui sì presso già fuste, ohimé, per cader nel profondo, dov'ha l'aer mai sempre oscuro velo, e ricovrate la vita, e voi stesso, drizzando tutti a quel Signor giocondo i pensier vostri, che n'aspetta in Cielo.

Tempo è homai, poi che cangiate il pelo, che pensieri, e desir cangiar deveste, Varchi gentil, volgendogli da queste cose basse, e mortali a i ben del Cielo;

e quel Signor, per cui già caldo, e gielo in un medesimo tempo al cuore haveste, ${ }^{94}$ lasciate in tutto, e 'l santo Amor celeste meco seguite, pien d'ardente zelo.

Dall'uno harete dispiacere, e guerra, dall'altro sempremai diletto, e pace; 
quello è di biasmo, e questo è d'honor duce;

l'un giova poco, e l'altro sempre piace;

quel manda il corpo, e l'anima sotterra, quest'altro al Ciel per dritta via conduce.

La fiamma ch'io portai nel core, e porto, non che spenta giammai, del suo valore non scemò dramma, anzi crebbe a tutt'ore, e cresce ancora, e se val mio conforto mai sempre crescerà, ch'ad altro porto vele non volge mai chi brama onore, perch'io di lei mi lodo, e quel signore ringrazio umil che m'ha tanto alto scorto.

Ché ben so quanto è folle chi se stesso crede forte a tal lume, che gran pondo regger non può da sé picciolo stelo.

Non so qual già dite periglio espresso: veggio voi, dietro il volgo in basso fondo, mortal voglia chiamar celeste zelo.

Se bene io cangio d'ora in ora il pelo, non cangio mente mai, né voi dovreste, saggio, cercar di torcermi da queste cure che vivo altrui fanno ire al Cielo.

Quel caldo stesso, quello stesso gielo, ch'io provai sempre, or pruovo, e voi s'aveste scintille mai di vero amor celeste, ardete meco d'un medesmo zelo.

Non dee vostra credenza o l'altrui guerra farvi turbar la mia certezza e pace, né cieco offrirsi ad uom non losco duce.

Se la strada d'onor per me vi piace, o bramate per voi non star sotterra, l'amor ch'io seguo è quel ch'a ciò conduce.

L'occasione reale, che il primo sonetto grazziniano lascia intravedere all'origine dello scambio, consolida l'impressione di non convenzionalità che traspare da questi versi, il cui regime dialettico è assai distante dal tono univoco di pia persuasione su cui sono costruiti in maniera modulare gli altri sonetti con destinatario. La conferma si coglie nella sintassi e nel lessico, forgiati in un andamento contraddittorio, in cui risulta sensibilmente sottoesposto quel codice petrarchesco che, come si è visto, alimenta le rime spirituali grazziniane ed è largamente presente negli altri sonetti scambiati con il Varchi su argomenti 
profani. Di un netto cambio di prospettiva parlano le altre due coppie di sonetti, che mostrano la disponibilità del Varchi a trasferirsi sul terreno devoto peculiare della spiritualità dell'amico, rinunciando a quei valori terreni orgogliosamente propalati nei sonetti appena visti. Con particolare intensità ciò avviene in Lasca, altro Febo, altra Minerva omai, la cui disadorna scansione in interrogative retoriche esprime una dimensione di appressamento alla morte di tenore squisitamente penitenziale:

Lasca, altro Febo, altra Minerva omai, se stolti ambo non siam, cercar dovemo. Dunque, vecchi all'età, giovin saremo a sapere schivar gli ultimi guai?

Dunque vani farem notte e dì lai, ed al principio fia pari l'estremo? Dunque al più chiaro Sol ciechi non andremo, e suo dritto a Gesù non darem mai?

Dunque al tremendo dì non de' chiamati, ma sarem di color ch'al fuoco eterno fien tra pianti e stridori, oimé, dannati?

Dunque al Regno del Ciel, dunque a i beati spirti, dunque a chi fia sempre ab eterno, cangerem questo basso e breve inferno?

Franco Pignatti 
I. Degli avvertimenti della lingua sopra'l Decameron. Volume primo. Del cavalier Lionardo Salviati. Diviso in tre libri [...], In Venezia, MDLXXXIIII (col.: In Venetia, Presso Domenico, \& Gio. Battista Guerra, fratelli. MDLXXXIIII), p. Ios.

2. L'edizione, già pronta nel I799, fu pubblicata a Livorno nel I 8 I6, ma con la data I799, dal figlio del Poggiali, Domenico, dopo che il padre era morto nel i 8r 4. Su Leopardi possibile lettore del Grazzini pastorale ha scritto $\mathrm{N}$. Borsellino, A Silvia, variazioni su un sonetto pastorale, in « Paragone. Letteratura », XXVIII (1977), pp. 45-55.

3. Si legge in Antonfrancesco Grazzini, Le rime burlesche, a cura di C. Verzone, Firenze, Sansoni, r 882, pp. CXXI-CXXIV, e in R.J. Rodini, Antonfrancesco Grazzini poet, dramatist and novelliere. 1503-1584, Madison-Milwaukee-London, University of Wisconsin Press, 1970, pp. 165-71.

4. La descrizione in GraZZINI, Le rime burlesche, pp. LXI-LXII.

5. L'egloga è trasmessa, insieme con le altre nove, anche dal Magl. VII.r 240, cc. 36r-40r (sul quale cfr. Grazzini, Le rime burlesche, pp. LVII-LVIII) ed edita in M. Plaisance, Antonfrancesco Grazzini dit Lasca (I505-1584). Ecrire dans la Florence des Médicis, Manziana, Vecchiarelli, 2005, pp. II 3 -I 9 .

6. Cfr. Grazzini, Le rime burlesche, pp. LXVII-LXVIII.

7. Rime di Antonfrancesco Grazzini detto il Lasca, In Firenze, Nella Stamperia di Francesco Moücke, MDCCXXXXI- MDCCXXXXII, Parte prima, pp. XIII-XIV.

8. Antonfrancesco Grazzini, Orazioni alla croce, a cura di D. Moreni, Firenze, Per il Magheri, I 822, p. 20; si leggono poi in Le Cene ed altre prose di Antonfrancesco Grazzini detto il Lasca riscontrate sui migliori codici, a cura di P. Fanfani, Firenze, F. Le Monnier, I857, pp. 379-95 (rist. Firenze, Succ. Le Monnier, 1888).

9. Le Orazioni erano già state segnalate da Antonio Maria Biscioni nella sua Vita del Lasca (Rime di Antonfrancesco Grazzini detto il Lasca, Parte prima, cit., p. LVI), che indicava le tre del Magl. XXXV.44 e la quarta « appresso il nostro Stampatore »; poi dal Poggiali, senza notizie sui codici, in Serie de' testi di lingua stampati, che si citano nel Vocabolario degli Accademici della Crusca posseduta da Gaetano Poggiali, I, Livorno, T. Masi e Comp., I 8 I3, p. 179.

Io. Grazzini, Le rime burlesche, pp. LXI-LXII.

I I. Per una panoramica ci si può ancora rivolgere ad A. D'Ancona, Origini del teatro italiano, I, Torino, Loescher, I89I pp. 33 I-67.

I 2. F. Decroisette, Fêtes religienses, fêtes princières an XVI siècles: Les Médicis et la fête de l'Annonciation à Florence, in Culture et religion en Espagne et en Italie aux $X V^{e}$ et $X V T^{e}$ siècles, Abbeville, F. Paillart, I980, pp. I I-4I; Il luogo teatrale a Firenze. Brunelleschi, Vasari, Buontalenti, Parigi. Firenze Palazzo Medici Riccardi Museo Mediceo 31 maggio-3I ottobre 1975, a cura di M. Fabbri - E. Garbero Zorzi - A.M. Petrioli Tofani, Milano, Electa, 1975, pp. 62-69, in particolare pp. 67-68; N. Newbigin, Feste d'Oltrarno. Plays in Churches in fifteenth-century Florence, I, Firenze, Olschki, 1996, pp. I-43. Sulla rappresentazione del I 533 possediamo un'accurata descrizione degli apparati nel Diario dal 1437 al 1533 con altre memorie e le nozze del duca Alessandro (Firenze, Bibl. nazionale centrale, Nuovi acquisti 982), probabile opera di Iacopo di ser Guido d'Anghiari. Vale la pena di ricordare che nel 1532 Grazzini abitava con i fratelli Girolamo, Lorenzo e Simone in una casa con giardino in via delle Caldaie, nel popolo di S. Felice in Piazza, presso piazza S. Spirito. 


\section{Franco Pignatti}

I3. Il primo testo del genere è la Morte del Re Acab, rappresentata dalla Compagnia del Vangelista nel I 5 59; cfr. l'introduzione ai Drammi spirituali inediti di Giovanmaria Cecchi notaio fiorentino del XVI secolo, a cura di R. Rocchi, Firenze, Le Monnier, I 895-1900.

14. Grazzini gli indirizzò altri due sonetti caudati (Io vo' farvi saper, caro Bettino; Io t'avvertisco, caro mio Bettino, in Le rime burlesche, pp. 59-60, 63-64), pure di impianto epistolare, il secondo contenente una scherzosa vituperatio del destinatario, e la lettera del 20 agosto 1547 che descrive l'inondazione di Firenze e del Casentino avvenuta il giorno i 3 (Della inondazione di Firenze nel MDXLVII. Lettera inedita di Antonfrancesco Grazzini detto il Lasca, a cura di G.E. Saltini, Firenze, Stamperia sulle Logge del grano, I865 e in "Il Borghini», III, I865, pp. 36-43; poi in Antonfrancesco Grazzini, Scritti scelti in prosa e in poesia, a cura di R. Fornaciari, Firenze, Sansoni, i9i I, pp. r6 I-67). Il Bettini fu amico di Luca Martini, Lorenzo Scala, Giovan Battista Busini, Benedetto Varchi e di artisti fiorentini; in una lettera del 23 novembre I 546 Niccolò Martelli ne apprezza l'eleganza dello stile epistolare (Niccolò MarTelli, Dal primo e dal secondo libro delle lettere, a cura di C. Marconcini, Lanciano, Carabba, I916, pp. 98- IO2). Il 29 settembre I 545 fu accolto nell'Accademia Fiorentina. Su un cartone donato al Bettini da Michelangelo, verso il 1533 il Pontormo dipinse su tavola la celebre Venere e Cupido oggi alla Galleria dell'Accademia, destinata alla camera del Bettini nel palazzo di famiglia, ma il duca Alessandro s'impossessò della tela appena terminata. Il programma iconografico della stanza prevedeva inoltre la raffigurazione, entro lunette, dei poeti toscani che avevano cantato d'amore: Vasari (Le vite de' più eccellenti pittori, scultori ed architettori, a cura di G. Milanesi, VII, Firenze, Sansoni, I 906, p. 595) attesta che il Bronzino realizzò i ritratti di Dante, Petrarca e Boccaccio. A Roma il Bettini è attestato già nel I 537 (cfr. Benvenuto Celurin, Vita, in Opere di Baldassarre Castiglione, Giovanni Della Casa, Benvenuto Cellini, a cura di C. Cordié, Milano-Napoli, Ricciardi, I 960, pp. 687-88), nel I 544 era console della Nazione fiorentina (J. Delumeau, Vie économique et sociale de Rome dans la seconde moitié du XVIII siècle, I, Paris, E. De Bocard, 1957, p. 209 n.), ma è probabile che all'origine della lontananza da Firenze ci fosse l'ostilità del duca, dato che egli apparteneva alle famiglie fiorentine favorevoli alla Repubblica e aveva militato contro gli Spagnoli durante l'assedio (Benedetto Varchi, Storia fiorentina, in Opere di Benedetto Varchi ora per la prima volta raccolte, I, Trieste, Lloyd Austriaco, I 858, p. 303 b). Morì alla fine del I 55 I. Varchi gli dedicò nell'edizione torrentiniana del I 549 la Lezione nella quale si disputa quale sia più nobile arte la scultura, o la pittura, del marzo I 547 (un sonetto a lui diretto in Opere, cit., II, 1959, p. 838 b). Cfr. Annibal Caro, Lettere familiari, a cura di A. Greco, I, Firenze, Le Monnier, I957, pp. 299-300, 334; II, 1959, p. 66; Il carteggio di Michelangelo, ed. postuma di P. Poggi a cura di P. Barocchi e R. Ristori, IV, Firenze, S.P.E.S, I979, ad ind:; M. Plaisance, Une première affirmation de la politique culturelle de Côme I'r: la transformation de l'Académie des "Humidi" en Académie Florentine, in L'Accademia e il suo principe. Cultura e politica a Firenze al tempo di Cosimo I e di Francesco de' Medici, Manziana, Vecchiarelli, 2004, p. 37 n.; Id., Culture et politique à Florence de I542 à I55I. Lasca et les "Humidi" aux prises avec l'Académie Florentine, ibid., pp. 22 I-22; M. Firpo, Gli affreschi di Pontormo a San Loren₹o. Eresia, politica e cultura nella Firenze di Cosimo I, Torino, Einaudi, I997, p. 308 n.; R. Aste, Bartolomeo Bettini e la decorazione della sua "camera" fiorentina, in Venere e Amore. Michelangelo e la nuova bellezza ideale. Catalogo della mostra Firenze, Galleria dell' Accademia 26 gingno-3 novembre 2002, a cura di F. Falletti e J. Katz Nelson, Firenze, Giunti, 2002, pp. 2-25, e passim nel volume.

I s. Il sonetto è stato evidenziato da M. Plaisance, La diffusione a Firenze delle «Rime» di Vittoria Colonna, in ID., L'Accademia e il suo principe, cit., pp. 28 I-89; dopo di lui S. Bertelli, Egemonia linguistica come egemonia culturale e politica nella Firenze cosimiana, in "Bibliothèque d'Humanisme et Renaissance», XXXVIII (1976), p. 26I, e M. Firpo, Gli affreschi di Pontormo a San Lorenzo, cit., p. 177.

i6. Grazzini, Le rime burlesche, pp. 6o-62, vv. 9-59.

17. L'Ochino lasciò Ferrara dopo l'Epifania e lungo il cammino fu attivo sui pulpiti di Faenza - inizio di febbraio - e di Brisighella, i e I 2 febbraio (F. Lanzoni, La Controriforma 
nella città e diocesi di Faenza, Faenza, Stab. grafico F. Lega, 1925, pp. 50-51). Margherita d'Austria aveva ottenuto da papa Paolo III l'assicurazione di far predicare l'Ochino a Firenze nella Quaresima del I 538 con molto anticipo. Una lettera della duchessa di Firenze al pontefice a questo proposito data is ottobre 1537 (B. Nicolini, Sui rapporti di Bernardino Ocbino con le città di Bologna e Lucca, in «Atti dell'Accademia Pontaniana », VI, I956-57, pp. 9596 n. 8); nell'ottobre I 537, prima di recarsi a Ferrara, l'Ochino aveva predicato a Prato.

i 8. Vittoria Colonna, Carteggio, a cura di E. Ferrero - G. Müller, Torino, Loescher, i 889, pp. I 56-58: « Mirai in Ierusalem, diedi in Egitto, pensai starme a Bologna in silentio e il dì medesmo che dal Castello partii per andarvi hebbi risposta da madama qui che faceva predicar el padre in Pisa, non in Firenza; così voltai le redine, et per fugir cirimonie venni in un monesterio; [...] ma infin quando più godeva delle mirabil prediche, è stata tanta la instantia de Firenze che contra la voluntà de madamma ha bisognato remandarlo a quella città, et io ho concorso per lo honore de Dio et magior frutto. Me sto qui consolata sin che serrà tempo ir a Lucca a bagni. [...] El padre Belardin quando partì, che fu ieri, me disse che molto basava le man de V. Ex.tia, et se con le prediche che addesso non se n'è Vostra Ex.tia scordato, io spero che lo haverimo lì la quaresima che viene; certo qui per tutto è adorato, et son tutte burle, se non le sue prediche de quaresima ».

I 9. S. Adorni-Braccesi, "Una città infetta ». La Repubblica di Lucca nella crisi religiosa del Cinquecento, Firenze, Olschki, 1994, p. 94 n. I4I. La Adorni-Braccesi individua il motivo dell'invito nella preoccupazione dei maggiorenti lucchesi di procurarsi tramite la Colonna un'influenza sul cognato di lei, Alfonso d'Avalos marchese del Vasto, comandante degli eserciti spagnoli in Italia, allora impegnato nelle trattative per l'acquartieramento delle truppe nella penisola.

20. Ibid., pp. 94-95 n. I4I; cfr. L. von Pastor, Storia dei papi, V, Roma, Desclée e C., I924, p. I 85 .

2i. Colonna, Carteggio, pp. i 59-6r.

22. Adorni-Braccesi, "Una città infetta », cit., p. 95 n. I42.

23. In questa circostanza fu presente Giovanni Guidiccioni, che scrisse ad Annibal Caro a Roma di avere udito il cappuccino e di avere composto, ispirato dalle sue parole, due sonetti (Giovanni Guidiccioni, Le lettere, a cura di M.T. Graziosi, II, Roma, Bonacci, i979, p. io; la datazione della missiva si basa sulla lettera del Caro al Guidiccioni del 22 agosto I 938 , in cui Annibale dichiara di attendere il secondo sonetto, cfr. CARo, Lettere familiari, I, p. I I 5); i sonetti furono poi tre: $O$ messaggier di Dio, che 'n bigia vesta, A quei ferventi spirti, a le parole, $O$ sante figlie de l'eterno Sire (Giovanni Guidiccioni - Francesco Beccuti Coppetta, Rime, a cura di E. Chiorboli, Bari, Laterza, I9I 2, pp. 76-77). Cinque omelie lucchesi furono stampate a Venezia, per Bernardino de Viano, nel i 54I (nel colophon la data del 16 marzo), ora in Patterns of Perfection. Seven Sermons in Patria by Bernardino Ocbino (I487-I564), edited and introduced by Ph. McNair, Cambridge, Anastasia Press, 1999.

24. Colonna, Carteggio, p. $6_{3}$.

25. I processi inquisitoriali di Pietro Carnesecchi (I557-1567), a cura di M. Firpo - D. Marcatto, II, Il processo sotto Pio V (I566-I567), Città del Vaticano, Archivio Segreto Vaticano, 2000, t. 2, p. 429: «Dippoi la [V. Colonna] reviddi a Fiorenza essendo lei capitata in quelle bande per andare alle Bagne di Lucca dove, essendo andato ancor io per mia buona sorte in quel tempo medesimo, hebbi occasione di pigliare ancor più stretta familiarità et servitù con lei » (Io novembre I 566); t. 3, p. I073: «Mi ricordo essere stato alli Bagni di Lucca nel tempo medesimo che vi era la sudetta marchesa et d'haverla qualche volta visitata, ma non mi ricordo già d'essermi trovato in Lucca quando [vi fu] lei et d'esser mai stato in quella città 


\section{Franco Pignatti}

se non in quel anno per transito, tornandomene da detti bagni verso la patria. Et questo fu ne l'anno i 538 » (2 marzo i 567 ).

26. Cfr. M. Plaisance, Vittoria Colonna et l'Académie Florentine: un commentaire en forme d'oraison funèbre, in Id., L'Accademia e il suo principe, cit., pp. 291-309. Plaisance segnala tre lezioni di accademici su poesie della Colonna: Bernardo Canigiani sul sonetto D'ogni sua gloria fu largo al mio sole il 29 gennaio I 542 (pubblicata anonima in D. Tordi, Bricciche letterarie, Roma, Tip. Pallotta, I889); Francesco di Niccolò Bottegari sul sonetto Perché dal tauro l'infiammato corno il 22 novembre I 545; Pierfrancesco di Tommaso Ginori il I 3 luglio I 550.

27. Rime di Antonfrancesco Grazzini detto il Lasca, Parte prima, cit., p. 30; alla stessa circostanza risale il madrigale Voi mi darete i dolci eletti canti (ibid., p. I63), trasmesso, di mano di Grazzini e con correzioni autografe, dal ms. II.IV.249 della Biblioteca nazionale centrale di Firenze (c. Ios r-v), che però Verzone assegna a Vincenzo Sbarri, amico del Lasca (Grazzini, Le rime burlesche, pp. CII-CIII).

28. Cfr. Vittoria Colonna, Rime, a cura di A. Bullock, Bari, Laterza, i982, pp. 224-29, p. 258 , p. 260 .

29. Alcuni episodi riporta R.H. Bainton, Bernardino Ocbino esule e riformatore senese del Cinquecento 1487-1563, Firenze, Sansoni, 1940, pp. 33-38.

30. Cc. 8ov-82r; seguono nel codice, sempre di Grazzini «Una canzone cantata nel detto luogo de' notai " (cioè recitata dai notai venuti apposta da Firenze) e una licentia «nella fine del desinare » (cc. 82v-83v; i tre testi sono editi in Rime di Antonfrancesco Grazzini detto il Lasca, Parte seconda, cit., pp. I०8-10, pp. 227-28). I primi due componimenti alludono a una qualche questione sorta tra la Compagnia e l'Ordine dei notai. Per la Cicilia Iacopo Del Polta da Bientina compose l'interconvivio Dell'inganno, contro i frati del vicino convento di S. Francesco che tentavano di appropriarsi di un terreno di proprietà della Compagnia (M. Cataudella, Jacopo da Bientina e un suo interconvivio, in "Filologia romanza », VII, I960, pp. I43-56; Id., Farse morali forentine, testi raccolti e annotati da M. Montanile, Salerno, Edisud, I984, pp. I Io-2 I). Alla Cicilia è destinato anche il Canto dei contadini Alfonso de' Pazzi, probabilmente del i 547 (A. Castellani, Nuovi canti carnascialeschi di Firenze. Le "Canzone" e mascherate di Alfonso de' Pazzi, Firenze, Olschki, 2006, pp. 25 8-60). Uno scorcio sull'assetto della Cicilia offre una lettera di N. Martelli del 7 aprile I 546 ad Alessandro Davanzati, che ne era quell'anno governatore per la terza volta e al contempo era governatore anche della Compagnia del Bechello: nella Cicilia «concorrono una nobiltà infinita di forse 500 persone: e imprima gli offitii del culto divino con divotione (in detto luogo) sempre havete fatti osservar et solennemente cantare con le musiche et con le commedie honeste secondo la qualità de' tempi: e 'l pasto intra i fratelli che si costuma di fare sono stati bene ordinati (con parsimonia però) per non esser biasimati: ma con una pace et quiete mirabilissima che non vi è stata poca lode perché dove è moltitudine sempre suole esser confusione e imbriacherie et questioni e al tempo vostro non mai che meritereste per questa virtù governare una cità non ch'una Cicilia » (Il primo libro delle lettere di Niccolò Martelli, Firenze, In Fiorenza, a instanza dell'Auttore [A. Doni], l'anno mDXLVI a dì XvIII del Mese di Giugno, I 546, pp. 86-87).

3I. Grazzini, Le rime burlesche, pp. 2 I0-I 4, pp. 2 I 7-I 9.

32. Grazzini dedica alcune ottave alla Compagnia di S. Sebastiano, che metteva in scena spettacoli teatrali (GrazzinI, Le rime burlesche, pp. 426-27).

33. Grazzini, Le rime burlesche, p. 482, vv. 35-36.

34. Grazzini, Le rime burlesche, p. 53, son. LXIV, vv. 7-8. 
35. P. Simoncelli, L'evangelismo italiano del Cinquecento: questione religiosa e nicodemismo politico, Roma, Istituto storico italiano per l'età moderna, I979, p. 334, p. 346, p. 385 .

36. Alle Orazioni si sono finora interessati Plaisance, Vittoria Colonna et l'Académie Florentine, cit., pp. 283-84; Firpo, Gli affreschi di Pontormo a San Lorenzo, cit., pp. 177-78.

37. Il Bembo doveva allora già avere incassato le condanne di Ambrogio Catarino Politi nel Compendio d'errori, et inganni luterani, contenuti in un libretto, senza nome de l'autore, intitolato Trattato utilissimo del benefitio di Christo crucifisso (Roma, Nella contrada del Pellegrino, I 544), di Galeazzo Florimonte al concilio di Trento il 2 I luglio i 546 e l'inclusione della traduzione francese (Lione, Jean de Tournes, I 545) nell'Indice della Sorbona del I 547 (cfr. Benedetto Da Mantova, Il beneficio di Cristo con le versioni del secolo XVI. Documenti e testimonianze, a cura di S. Caponetto, Firenze-Chicago, Sansoni-The Newberry Library, 1972, pp. 343-422, pp. 439-40; Index de l'Université de Paris I544, I545, I547, I549, I55I, I556, a cura di J.M. De Bujanda - F.M. Higman - J.L. Farge, Sherbrooke-Genève, Editions de l'Université de Sherbrooke - Droz, I985, p. 485 ).

38. Il sermone del Varchi fu publicato da L. Torrentino nello stesso i 549 insieme con l'Orazione funerale per la madre di Cosimo I, Maria Salviati, pronunciata dal Varchi in Accademia il I6 dicembre I 543. La tempestività della stampa non deve ingannare: il Varchi fece precedere il sermone da una lettera ad Alessandro Lenzi, in cui dichiarava le sue perplessità sul contenuto teologico dello scritto e scaricava sul tipografo la responsabilità della sua diffusione a stampa (cfr. Simoncelli, L'evangelismo italiano, cit., pp. 333-34).

39. A proposito di questo passo M. Firpo ha parlato, un po' enfaticamente, di «ambigui scrupoli di ortodossia», rivelatori dell'attenzione «a restare sul terreno devozionale, ed evitare spinose questioni dottrinali » (Firpo, Gli affreschi di Pontormo a San Lorenzo, cit., p. I 7 8).

40. GrazZInI, Orazioni alla croce, pp. 3I-32.

4i. Antonfrancesco Grazzini, La Strega, édition critique avec introduction et notes par M. Plaisance, Abbeville, F. Paillart, 1976, pp. 26-35; Simoncelli, L'evangelismo italiano del Cinquecento, cit., pp. 360-61, pp. 374-75.

42. Cfr. E. Garavelli, Lodovico Domenichi e i 'Nicodemiana' di Calvino. Storia di un libro perduto e ritrovato, Manziana, Vecchiarelli, 2004.

43. Simoncelli, L'evangelismo italiano, cit., p. 38I.

44. Agli anni Quaranta pensa genericamente Firpo, Gli affreschi di Pontormo a San Loren₹o, cit., p. 178 .

45. GrazzInI, Orazioni alla croce, pp. 2, 4; anche Orazione quarta, p. 3 I, e l'Oratione exortatoria recitata per uno Romito, del I 540: «piglian l'essempio dal gran Re del Cielo / che 'n sulla croce steso a braccia aperte / aspetta et chiama ognuno a penitentia / per farlo poi del suo bel regno herede» (ms. II.IV.I, c. 82r).

46. Cfr. Antonfrancesco Grazzini, Commento di ser Niccodemo dalla Pietra al Migliaio sopra il Capitolo della salsiccia, a cura di F. Pignatti, in Ludi esegetici, prefazione di P. Procaccioli, I, Manziana, Vecchiarelli, 2004, pp. I 57-58, pp. 231-34.

47. Grazzini, Orazioni alla croce, p. 3.

48. Grazzini, Orazioni alla croce, p. 5.

49. Grazzini, Orazioni alla croce, p. 6. 
50. Grazzini, Orazioni alla croce, pp. 9-10.

5 I. Grazzini, Orazioni alla croce, p. I 3.

52. Grazzini, Orazioni alla croce, pp. i 5-i 6; Benedetto da Mantova, Il beneficio di Cristo, p. 5 6: "Questo è quello divino amore e carità, che nasce dalla fede non finta che inspira Dio alli suoi eletti, della quale dice san Paulo che opera per la carità. Ma perché la vita di Cristo, della cui imitazione ci dobbiamo vestire, fu una perpetua croce piena di tribulazioni, ignominie e persecuzioni, se vogliamo conformarci con la vita sua, ci bisogna portare di continuo la croce, come esso disse: "Se alcuno vorrà venire dopo me, disprezzi se stesso e tolga ogni giorno la croce sua e seguiti me"».

53. GrazZini, Orazioni alla croce, pp. io-I I.

54. Grazzini, Orazioni alla croce, pp. I I-I 2.

55. Per la localizzazione delle rime, si veda d'ora in poi la Tavola in appendice al presente lavoro.

56. Rime di Antonfrancesco Grazzini detto il Lasca. Parte seconda, cit., pp. 286-87: « Nel portarsi a Firenze solennemente la miracolosa Tavola dov'è l'effigie di Maria Santissima dell'Impruneta, il dì I 8 di ottobre I 530 per implorare la pioggia». Le funzioni tradizionali della Vergine erano quelle di evitare la siccità e di scongiurare le alluvioni, ovvero di suggellare paci, alleanze e acquisti territoriali. Poiché si riteneva che la Madonna non volesse albergare nella città, la tavola di Nostra Signora era portata a Firenze per la durata della cerimonia, o altrimenti la processione lambiva il territorio cittadino, costeggiando la cinta muraria o percorrendo i colli circostanti. Nell'ottobre i 529 , tuttavia, nella fase finale della Repubblica, per mettere al sicuro l'immagine dalle rapine e dai sacrilegi delle soldatesche imperiali, il governo l'aveva fatto portare a Firenze e l'aveva collocata in S. Maria del Fiore, nella cappella di S. Zenobi (VARCHI, Storia fiorentina, p. 2 I 6 a-b). Qui era stata oggetto di fervente devozione popolare come protettrice della città assediata, legando così la sua immagine alla causa repubblicana. Per questo motivo durante il governo di Cosimo I il culto fu diradato e la tavola fu portata a Firenze soltanto in due occasioni, nel I 538 e nel I 547, in entrambi casi per motivi legati alla regolazione delle acque (cfr. S. Mantini, Lo spazio sacro della Firenze medicea. Trasformazioni urbane e cerimoniali pubblici tra Quattrocento e Cinquecento, Firenze, Loggia de' Lanzi, I 995, pp. I70-80). Ora, già il buon senso da solo induce a dubitare della data che reca l'edizione settecentesca, ottobre I530: il io agosto i 530 era terminato l'assedio e appena l'8 ottobre l'esercito imperiale aveva lasciato il campo nei dintorni della città. La cronologia necessita di una correzione, che si ricava dalla medesima Storia fiorentina di Varchi: "Alla fine di febbraio [I 53 I] la tavola dell'Impruneta, la quale s'era riportata a' diciotto d'ottobre, si fece venire a Firenze, non perché non piovesse, come suole il più delle volte, ma perché piovesse » (VARCHI, Storia fiorentina, p. 336 b). Dunque il i 8 ottobre I 530 è il giorno in cui l'immagine in aura di repubblicanesimo era stata frettolosamente rimossa da Firenze e ricollocato nel santuario, da dove nel febbraio successivo, ancora i 530 secondo il calendario fiorentino $a b$ incarnatione, era stata portata in città per propiziare la pioggia, secondo la sua funzione tradizionale legata alla meteorologia, e per risollevare la cittadinanza stremata dall'assedio e preoccupata per un raccolto che si annunciava assai povero, come in effetti fu. A questa circostanza si dovrà ricondurre la coppia di sonetti grazziniani, sempre che si decida di dare credito alla notizia dell'edizione Moüke.

57. Plaisance, La diffusione a Firenze delle «Rime» di Vittoria Colonna, cit., p. 280.

58. Sulla mariologia ochiniana E. Campi, Michelangelo e Vittoria Colonna. Un dialogo artisticoteologico ispirato da Bernardino Ochino e altri saggi di storia della Riforma, Torino, Claudiana, I994, pp. 33-37. 
59. Riporto i testi nella redazione del Magliabechiano, segnalando le varianti e gli interventi editoriali.

6o. Più persuasiva la lezione dell'ed. Moücke: «stette ben per tre anni il ciel serrato ».

6r. Magl.: «prego ».

62. Lo stesso sintagma nel sonetto I.I della raccolta del ms. II.IV.I.

63. Magl.: « in core».

64. È clausola petrarchesca: Rvf. 26, 8 e Trimmphus Eternitatis I 40, anche Rvf. 107, 2; Rvf. 300 , 4.

65. Rime di Antonfrancesco Grazzini detto il Lasca, cit., pp. 286-87: « Per lo solennissimo ingresso fatto nella Città di Firenze il dì 18 novembre dell'anno I 547 della portentosa Tavola di Maria gloriosissima dell'Impruneta, acciocché c'impetri la serenità dell'aria ». Dell'evento conserva memoria il Diario forentino di Agostino Lapini dal 252 al 1596, a cura di O. Corazzini, Firenze, Sansoni, I 900, p. I06, il quale tramanda che la processione ebbe buon esito; siamo a poca distanza dall'alluvione del I 3 agosto descritta nella lettera a B. Bettini, di cui si è detto sopra, n. I4: Lapini parla infatti di piogge quasi ininterrotte che duravano da tre mesi. Questi due sonetti meteorologici, insieme con gli altri due presenti nell'edizione Moüke, erano già stati editi in Memorie istoriche della miracolosa immagine di Maria Vergine dell'Impruneta raccolte da Giovambattista Casotti lettore d'Istoria sacra e profana nello Studio di Firenze, Firenze, G. Manni, 1714, pp. 22-23.

66. Magl.: «tue».

67. Magl.: «Volgi, volgi omai gli al tuo bell'Arno».

68. Così giudica anche Plaisance, La diffusione a Firenze delle «Rime» di Vittoria Colonna, cit., p. 282: «forse questi sonetti legati alla meteorologia fiorentina si potevano difficilmente attribuire alla marchesa di Pescara ».

69. Al I 54 I li riporta senz'altro Plaisance nell'introduzione della Strega di Grazzini (ed. cit., p. 30).

70. Manca anche il sonetto inserito nella seconda Orazione alla croce, il quale però, a parte la sede particolare che lo distingue, è un testo di singolare rozzezza se confrontato con il resto delle rime spirituali.

7I. Il sonetto è preceduto dalla didascalia «Nel venerdì santo ».

72. Il testo dei dodici sonetti è, salvo corruttela, quello del ms. II.IV.I; dove necessario, indico e discuto le varianti del Magliabechiano.

73. Campi, Michelangelo e Vittoria Colonna, cit., pp. 22-33.

74. Grazzini, Orazioni alla croce, pp. I 3-I4.

75. Cfr. Rvf. 1 22, 5; Rvf. 195, 1; Rvf. 277, г4; Rvf. 316, 9-10; Rvf. 33 1, 60; Rvf. 360, 41; Rvf. 362,8 .

76. La perifrasi sta a indicare la Madonna. 


\section{Franco Pignatti}

77. M. Mazzetti, La poesia come vocazione morale: Vittoria Colonna, in «La Rassegna della letteratura italiana », LXXVII (I 973), pp. 57-99, p. 75.

78. Nel sonetto Non più, madre Maria, non più, raffrena, che è una consolatio rivolta dall'arcangelo Gabriele alla Madonna per la morte del figlio, v. I 3 : «sposa di Dio, figliuola e madre ».

79. Alla nota riservo le varianti del Magliabechiano: v, 2: «Vergine bella, le mie preci ascolta »; 4: « errando se ne va sola e pensosa »; x, 3: «Vergine santa, il cui valor pregiato ». Dove noteremo l'alternanza speculare nei testimoni di bella e santa, e la rimozione nel ms. II.IV.I del petrarchismo eclatante di v, 4 (cfr. Rvf. 35 , I) con un sintagma più discreto, e forse tuttavia memore de «l'alma dubbiosa e vaga» di $R v f$. i 25, 65.

8o. Ms. II.IV.r: «pena ».

8r. Magl.: «gli error».

82. Plaisance, La diffusione a Firenze delle «Rime» di Vittoria Colonna, cit., p. 283.

83. Rime di Antonfrancesco Grazzini detto il Lasca, Parte prima, cit., pp. 288-89.

84. Due ottave al Cini in Grazzini, Le rime burlesche, p. 4i 5.

85. Cfr. G. Bacchi, Il Monastero di "S. Elisabetta delle Convertite" di Firenze, in "Bollettino storico agostiniano», VII (193 I), pp. I45-47, 234-38. Una legge del i9 settembre I 553 stabiliva che le meretrici che facessero testamento destinassero I/4 delle loro sostanze al monastero; in caso di eredità ab intestato l'intero patrimonio doveva andare alle monache $\mathrm{e}$ se i beni superavano il loro fabbisogno erano devoluti allo Spedale degli Innocenti. Un rescritto ducale del 5 luglio I559, in risposta a una supplica delle monache, estese il privilegio alle meretrici non descritte, purché fossero di pubblica e mala fama (Legislazione toscana raccolta e illustrata dal dottore Loren₹o Cantini, Firenze, Stamp. Albizziniana da S. Maria in Campo, I 803, II, pp. 32 I-28; III, pp. 302-303; VIII, pp. 2 I 2-I 3). Nello Stato della Chiesa questa legge esisteva dal i 520 (Bullarium Romanum, Leone $X, \mathrm{~V}, 745$ ), la misura di I/4 era stata fissata da Clemente VII nel i 525 (ibid., Clemente VII, VI, 93).

86. Firenze, Bibl. nazionale centrale, Magl. VII.898, c. 191 r; Rime di Antonfrancesco Grazzini detto il Lasca, Parte prima, cit., p. 348. Giovanni da Pistoia ( I 509-I 578 ), notaio di professione, fu cancelliere dell'Accademia Fiorentina nel i 54 I e i 542 , e ammesso come accademico nel I 546 ; compose la commedia La gioia, rappresentata in Palazzo Vecchio nel i 50 e stampata a Venezia del 1586.

87. Firenze, Bibl. nazionale centrale, II.IX.45, c. 32v. Su Giulio Scala, speziale, morto nel I 585 , cfr. VASARI, Le vite de' più eccellenti pittori, scultori ed architettori, cit., IV, pp. 465-66. Il Lasca lo nomina in una madrigalessa in rapporto alla sua professione e un'altra gli dedica a proposito di certi suoi amori con una cortigiana (GrazzinI, Le rime burlesche, pp. 284, pp. 295-96; anche pp. 490-91).

88. GraZZINI, Le rime burlesche, p. 444, vv. 57-58; di Giulia, quando era in attività, Grazzini aveva cantato l'avvenenza nella madrigalessa Come chi pensa e crede, composta per Bernardo Ulivi, innamorato della donna (ibid., pp. 274-76); un'altra madrigalessa all'Ulivi sullo stesso tema ibid., pp. 276-77.

89. De' sonetti di M. Benedetto Varcbi colle risposte, e proposte di diversi. Parte seconda, In Fiorenza, Appresso Lorenzo Torrentino, MDLVII, p. 95; Opere di Benedetto Varchi, cit., II, p. 939 a-b.

90. De' sonetti di M. Benedetto Varchi colle risposte, e proposte di diversi. Parte seconda, cit., p. 96; Opere di Benedetto Varchi, cit., II, p. 939 b. 


\section{Antonfrancesco GrazZini}

91. Sonetti spirituali di M. Benedetto Varchi. Con alcune risposte, et proposte di diversi eccellentissimi ingegni, In Fiorenza, Nella Stamperia de' Giunti, I 573, p. 72; il sonetto è dato come propositivo di quello grazziniano stampato a p. ı०4, nonostante la discordanza rimica, che induce l'editore ottocentesca a pubblicarlo a sé (Opere di Benedetto Varchi, cit., II, p. 999 b). Il contenuto delle due poesie è in effetti compatibile, ma il dubbio permane.

92. Sonetti spirituali di M. Benedetto Varchi, cit., p. I I 8.

93. Firenze, Biblioteca Nazionale Centrale, Banco Rari 59, n. 7; Rime di Antonfrancesco Grazzini detto il Lasca, Parte prima, cit., p. 39; posteriore alla scarcerazione anche il sonetto Poiché allimmenso tuo sommo valore (ibid. p. 69; Banco Rari 59, n. 4), composto come auspicio per la guarigione di Del Rosso da una malattia. Sul personaggio cfr. P. Simoncini, Il cavaliere dimezzato: Paolo Del Rosso, fiorentino e letterato, Milano, F. Angeli, I990.

94. Su questo ossimoro, quale cellula caratteristico del petrarchismo, ha discorso a proposito di Grazzini, R. Gigliucci, Breve dolcezza, lungo malanno. Parodie dei contrapposti petrarcheschi, in Cum notibusse et comentaribusse. L'esegesi parodistica e giocosa del Cinquecento. Seminario di letteratura italiana Viterbo, 23-24 novembre 200I, a cura di A. Corsaro e P. Procaccioli, Manziana, Vecchiarelli, 2002, pp. 199-206. 


\title{
Franco Pignatti
}

\section{Poesie spirituali di Antonfrancesco Grazzini ${ }^{1}$}

\author{
Magl. VII.ı 29, \\ Ed. Moücke \\ Magl. II.IV. I
}

SONETTI

$A$ che più desiar terra, o tesoro? ${ }^{2}$

A questo Mondo rio, che sé non tiene $e^{3}$

Adunque io vi conforto e vi consiglio 4

Abi quanto è presto, e come a fuggir leve

Altro nuovo disio mi preme, e punge

Come già nel deserto bumilemente

Com'è, Varchi, di nuovo in voi risorto'

Con quella alma pietà dolce infinita

Cotal sento dolor gravoso, e forte

Deh dolce signor mio, signor clemente

Dov'è la tua superbia? ove son hora ${ }^{6}$

Dunque, giusto Signore, i vizi nostri

$\dot{E}$ questo quel sagrato legno, e santo

Ecco, Donna del Ciel, ch'bumile, e lieta

Fabio, che 'n Ciel vederti ancor disia'

Giammai non credett'io da gl'empi, e feri

Giulia, che 'l Mondo t'hai goduto, e bora ${ }^{8}$

Hoggi ch'ba'l sole $i$ bei lucenti rai

Hor che dagli occhi miei scquarciato è'll velo

Hor che dal Mondo, e dal suo cieco honore

Hor vegg'io ben, Signor, che chi si fida

Madre del vero Dio, figliuola, e sposa

Mirate, Martel mio, come repente?

\begin{tabular}{|c|c|}
\hline & $\begin{array}{l}\text { I, } 5 \\
\text { I, } 6\end{array}$ \\
\hline $2 \mathrm{I} 2 \mathrm{~V}, 2 \mathrm{I} 6 \mathrm{r}$ & II, $277-78$ \\
\hline 204V, I०4r & II, 237 \\
\hline $23 \mathrm{IV}$ & II, $286-87$ \\
\hline $208 v$ & $\mathrm{I}, 4$ \\
\hline $2 \mathrm{I} 4 \mathrm{~V}$ & II, 283 \\
\hline $203 \mathrm{r}, \mathrm{IO} 3 \mathrm{r}$ & $\mathrm{I}, 3$ \\
\hline IOOV & II, 235 \\
\hline \multicolumn{2}{|l|}{$2 \mathrm{IIr}$} \\
\hline $2 \mathrm{I} 7 \mathrm{r}$ & II, 284 \\
\hline $2 \mathrm{IIV}, \mathrm{IOIV}$ & II, 236 \\
\hline $232 \mathrm{r}$ & II, 287 \\
\hline $2 \mathrm{I} 9 \mathrm{~V}$ & II, $28 \mathrm{I}-82$ \\
\hline $204 \mathrm{r}, \mathrm{IO} 3 \mathrm{~V}$ & II, 238 \\
\hline $2 \mathrm{Igr}$ & II, 28 I \\
\hline $202 \mathrm{r}, \mathrm{IOI}$ & $\mathrm{I}, 2$ \\
\hline $203 \mathrm{~V}$, Ioor & $\mathrm{I}, 2$ \\
\hline $2 \mathrm{I} 2 \mathrm{r}$ & II, 278 \\
\hline $2 \mathrm{I} 8 \mathrm{r}, \mathrm{IO} \mathrm{r}$ & $\mathrm{I}, \mathrm{I}$ \\
\hline $230 \mathrm{~V}, \mathrm{IO} 2 \mathrm{r}$ & II, $237-38$ \\
\hline 2 Ior & II, 279 \\
\hline
\end{tabular}

Magl. VII. I०29,

Ed. Moücke

Magl. II.IV.I

Non più, Madre Maria, non più, raffrena

Poi che sempre tornar veggo di gielo

Poi che tra le ricchezze, e glorie bumane ${ }^{\mathrm{IO}}$

Pura luce infinita, almo splendore

Qual di rabbiosa tigre o di crud'angue

Qual più grave, o maggiore, empio peccato

Quelle piaghe, Signor, ch'io veggio scorte

Questi occhi, e questi piedi, che mi fanno

Saria forse giammai questo il secondo

Se mai dentro $i$ superni santi chiostri

Se per le colpe del popolo ingrato

Sendo voi nell'etade ancora acerba ${ }^{\mathrm{I}}$

Signor, s'io son d'un Angel tuo terreno

S'io veggo certo, e conosco il mio bene ${ }^{\mathrm{I} 3}$

\begin{tabular}{|c|c|}
\hline $209 \mathrm{r}$ & II, 285 \\
\hline $2 \mathrm{I} 6 \mathrm{~V}$ (mutilo)-2I $7 \mathrm{~V}, \mathrm{I} O 2 \mathrm{~V}$ & II, $236-37$ \\
\hline $2 \mathrm{I} 8 \mathrm{v}$ & II, $280-8 \mathrm{I}$ \\
\hline $233 \mathrm{r}, \mathrm{IO} 4 \mathrm{~V}$ & II, 238 \\
\hline $202 \mathrm{~V}, 105 \mathrm{r}$ & II, $235-36$ \\
\hline $209 \mathrm{~V}$ & II, $284-85$ \\
\hline $2 \mathrm{I} 5 \mathrm{r}$ & II, $283-84$ \\
\hline $2 \mathrm{IOV}$ & II, $276-77$ \\
\hline $2 \mathrm{I} 4 \mathrm{r}$ & II, $282-83$ \\
\hline $232 \mathrm{~V}$ & II, $287-88$ \\
\hline $23 \mathrm{Ir}$ & II, 286 \\
\hline $206 \mathrm{r}$ & II, $279-80$ \\
\hline $206 v^{12}$ & II, 276 \\
\hline $15 \mathrm{~V}$ & II, $278-79$ \\
\hline
\end{tabular}




\section{Antonfrancesco GrazzinI}

Temp'è, Varchi, hoggimai ch'affatto il core ${ }^{\mathrm{I}}$ Temp'è bene hoggimai ch'affatto il core ${ }^{\mathrm{Is}}$ Tempo è homai, poi che cangiate il pelo ${ }^{\mathrm{I} 6}$

$205 \mathrm{r}$

$208 \mathrm{r}$

$2 \mathrm{I} 3 \mathrm{r}$

23 or

$2 \mathrm{I} 3 \mathrm{~V}^{\mathrm{I}} 8$

$205 \mathrm{~V}$

MADRIGALI

Gioisce piucché mai lieto e giocondo ${ }^{20}$

Guarda il tuo gregge umile

Volgete gli occhi, disiosi, e 'ntenti ${ }^{\text {I9 }}$
I, 5

I, 3

II, 277

II, 282

I, 4

II, 280

Magl. VII. I०29

OTTAVE

Una Donna, Signor, ch'è tua fattura

$207 \mathrm{r}$

Dunque questo mio amor, sagro e divino

$207 \mathrm{~V}$

Ma perché cieco sono, infermo, e frale

$207 \mathrm{~V}$

O Re del Ciel, cui nulla circonscrive

$207 \mathrm{r}$

EGLOGA

Resterio, o là. Che pensi? o là Resterio ${ }^{21}$

$220 \mathrm{r}-226 \mathrm{~V}$

II, 306-I 2

Ed. Moücke

I, I 49

SONETTI SPIRITUALI NEL MS. II.IV.I, cc. IOOr-IO5r
I. Hor che da gl'occhi miei squarciat'è 'l velo
II. Deb dolce signor mio, signor clemente
III. Hoggi c'ba'll sole $i$ bei lucenti rai
IV. É questo quel sacrato legno, e santo
v. Madre del vero Dio, figliuola, e sposa
VI. Poi che sempre tornar veggio di gielo
vII. Cotal sento dolor gravoso, e forte
VIII. Già mai non credett'io da gl'empi, e feri
IX. Altro nuovo desio mi preme, e punge
x. Pura luce infinita, almo splendore
XI. Qual di rabbiosa tigre, o di crud'Angue
XII. Hor veggio ben, Signor, che chi si fida 


\section{Franco Pignatti}

I. Nei casi di discordanza tra i testimoni è adottata, salvo indicazione contraria, la grafia del ms. Magliabechiano.

2. Grazzini, Orazioni alla croce, p. 20.

3. A Lorenzo Scala. Il responsivo, Amor, Lasca gentil, da cui mi viene, in Rime di Antonfrancesco Grazzini detto il Lasca, Parte prima, cit., pp. 288-89.

4. Eidem.

5. De' sonetti di M. Benedetto Varcbi colle risposte, e proposte di diversi. Parte seconda, cit., p. 95; Opere di Benedetto Varchi, cit., II, p. 939 a.

6. Escluso dall'edizione Moüke a causa del contenuto violentemente anticlericale, il sonetto è inedito.

7. A Giulia Napoletana.

8. Eidem.

9. A Guglielmo Martelli.

Io. A Laura Battiferri.

I I. A Vincenzo Alamanni.

I 2. Corretto su O Dio, s'io son d'un Angel tuo terreno.

I3. A Giovambattista Cini.

14. Firenze, Biblioteca Nazionale Centrale, Banco Rari 59, n. 3; Sonetti spirituali di M. Benedetto Varchi, cit., p. I I 8; Opere di Benedetto Varchi, cit., II, p. 939 b.

I s. L'intitolazione «A Ms. Benedetto Varchi» è stata erasa e sovrascritta con «Al Cavalier Fra Pagol del Rosso ».

I6. A Benedetto Varchi; Banco Rari 59, n. 2; De' sonetti di M. Benedetto Varchi colle risposte, e proposte di diversi. Parte seconda, cit., p. 96; Opere di Benedetto Varcbi, cit., II, p. 939 b.

17. Banco Rari 59, n. 5; Sonetti spirituali di M. Benedetto Varchi, cit., p. I04.

18. Reca la lezione erronea Varchi, dunque nei lacci, e negli inganni.

I9. A Bastiano Antinori.

20. «Nella natività di s. Giovan Battista ».

21. Magl. VII.I 240, cc. 36r-40r; Plaisance, Antonfrancesco Grazzini dit Lasca (1505-1584), cit., pp. II3-I9. 